\title{
Novel Evolutionary Lineages Revealed in the Chaetothyriales (Fungi) Based on Multigene Phylogenetic Analyses and Comparison of ITS Secondary Structure
}

\author{
Martina Réblováa ${ }^{1 *}$, Wendy A. Untereiner ${ }^{2}$, Kamila Réblová ${ }^{3}$ \\ 1 Department of Taxonomy, Institute of Botany, Academy of Sciences, Průhonice, Czech Republic, 2 Department of Biology, Brandon University, Brandon, Manitoba,
} Canada, 3 Central European Institute of Technology, Masaryk University, Brno, Czech Republic

\begin{abstract}
Cyphellophora and Phialophora (Chaetothyriales, Pezizomycota) comprise species known from skin infections of humans and animals and from a variety of environmental sources. These fungi were studied based on the comparison of cultural and morphological features and phylogenetic analyses of five nuclear loci, i.e., internal transcribed spacer rDNA operon (ITS), large and small subunit nuclear ribosomal DNA (nuc28S rDNA, nuc18S rDNA), $\beta$-tubulin, DNA replication licensing factor ( $m c m 7)$ and second largest subunit of RNA polymerase II (rpb2). Phylogenetic results were supported by comparative analysis of ITS1 and ITS2 secondary structure of representatives of the Chaetothyriales and the identification of substitutions among the taxa analyzed. Base pairs with non-conserved, co-evolving nucleotides that maintain base pairing in the RNA transcript and unique evolutionary motifs in the ITS2 that characterize whole clades or individual taxa were mapped on predicted secondary structure models. Morphological characteristics, structural data and phylogenetic analyses of three datasets, i.e., ITS, ITS- $\beta$-tubulin and 28S-18S-rpb2- $\mathrm{mcm} 7$, define a robust clade containing eight species of Cyphellophora (including the type) and six species of Phialophora. These taxa are now accommodated in the Cyphellophoraceae, a novel evolutionary lineage within the Chaetothyriales. Cyphellophora is emended and expanded to encompass species with both septate and nonseptate conidia formed on discrete, intercalary, terminal or lateral phialides. Six new combinations in Cyphellophora are proposed and a dichotomous key to species accepted in the genus is provided. Cyphellophora eugeniae and C. hylomeconis, which grouped in the Chaetothyriaceae, represent another novel lineage and are introduced as the type species of separate genera.
\end{abstract}

Citation: Réblová M, Untereiner WA, Réblová K (2013) Novel Evolutionary Lineages Revealed in the Chaetothyriales (Fungi) Based on Multigene Phylogenetic Analyses and Comparison of ITS Secondary Structure. PLoS ONE 8(5): e63547. doi:10.1371/journal.pone.0063547

Editor: Steven Harris, University of Nebraska, United States of America

Received January 11, 2013; Accepted April 1, 2013; Published May 28, 2013

Copyright: (C) 2013 Réblová et al. This is an open-access article distributed under the terms of the Creative Commons Attribution License, which permits unrestricted use, distribution, and reproduction in any medium, provided the original author and source are credited.

Funding: This work was supported by the Grant Agency of the Czech Republic (GAP 506/12/0038; www.gacr.cz). Additional support was provided to MR by a long-term research development project of the Institute of Botany, Academy of Sciences (RVO 67985939). KR was funded by the Project CEITEC - Central European Institute of Technology from European Regional Development Fund (CZ.1.05/1.1.00/02.0068; www.ceitec.eu). WAU was supported by a Discovery Grant from the Natural Science and Engineering Research Council of Canada (www.nserc-crsng.gc.ca) and by infrastructure awards from the Canada Foundation for Innovation. The funders had no role in study design, data collection and analysis, decision to publish, or preparation of the manuscript.

Competing Interests: The authors have declared that no competing interests exist.

* E-mail: martina.reblova@ibot.cas.cz

\section{Introduction}

The anamorphic Herpotrichiellaceae (Chaetothyriales) comprise morphologically diverse dematiaceous fungi that include both saprobic (i.e., non-pathogenic) and medically important species. The latter are involved in long-term infections of skin and subcutaneous tissue of humans and animals that include chromatoblastomycoses and phaeohyphomycoses [1,2,3,4,5]. Members of the order characterized by a lower degree of virulence or suspected to cause infections have been isolated from subcutaneous tissue, nail or skin scrapings [5,6,7]. A number of these taxa, which are described in the anamorph genera Cyphellophora G.A. de Vries and Phialophora Medlar, are of uncertain position within the Chaetothyriales.

Cuphellophora, originally introduced for a single species C. laciniata G.A. de Vries [6], encompasses dematiaceous fungi with septate, branched hyphae, intercalary, terminal or lateral phialides with indistinct or funnel-shaped collarettes, and septate, hyaline or pale brown conidia ranging in shape from oblong to fusiform or vermiform. Of the twelve members of the genus described to date, five were isolated samples of animal origin (i.e., nails and skin) $[4,6,8,9,10,11]$, whereas the other species occur in soil [12], on plants $[13,14]$, or as plant endophytes $[7,15]$. Based on the analysis of small subunit nuclear ribosomal DNA (nuc18S rDNA) sequences, five species of Cyphellophora formed a strongly supported monophyletic clade that was sister to representatives of the Herpotrichiellaceae [13]. In a multigene phylogeny [16], C. laciniata and Ceramothyrium carniolicum (Rehm) Petrak (Chaetothyriaceae) were inferred as sister to the Herpotrichiellaceae within the Chaetothyriales. Analysis of internal transcribed spacer rDNA operon (ITS), $\beta$-tubulin and large subunit nuclear ribosomal DNA (nuc28S rDNA) sequences subsequently resolved Cyphellophora and Phialophora as close relatives within the Chaetothyriales, although both genera were paraphyletic $[11,14,17,18]$. 
ITS maximum-likelihood tree (RAxML)

61 sequences of 36 species, 747 sites. Symbol 0 indicates branch support: BS/ML and BS/pP $=100 \%$ or 1.0

$\perp$ intercalary phialides
$\odot$ budding cells

T ex-type strain nonclinical strain (plants, soil, nutrient-poor substrates)

- clinical strain (human and animal infections)

- species with strains known from clinical and nonclinical sources

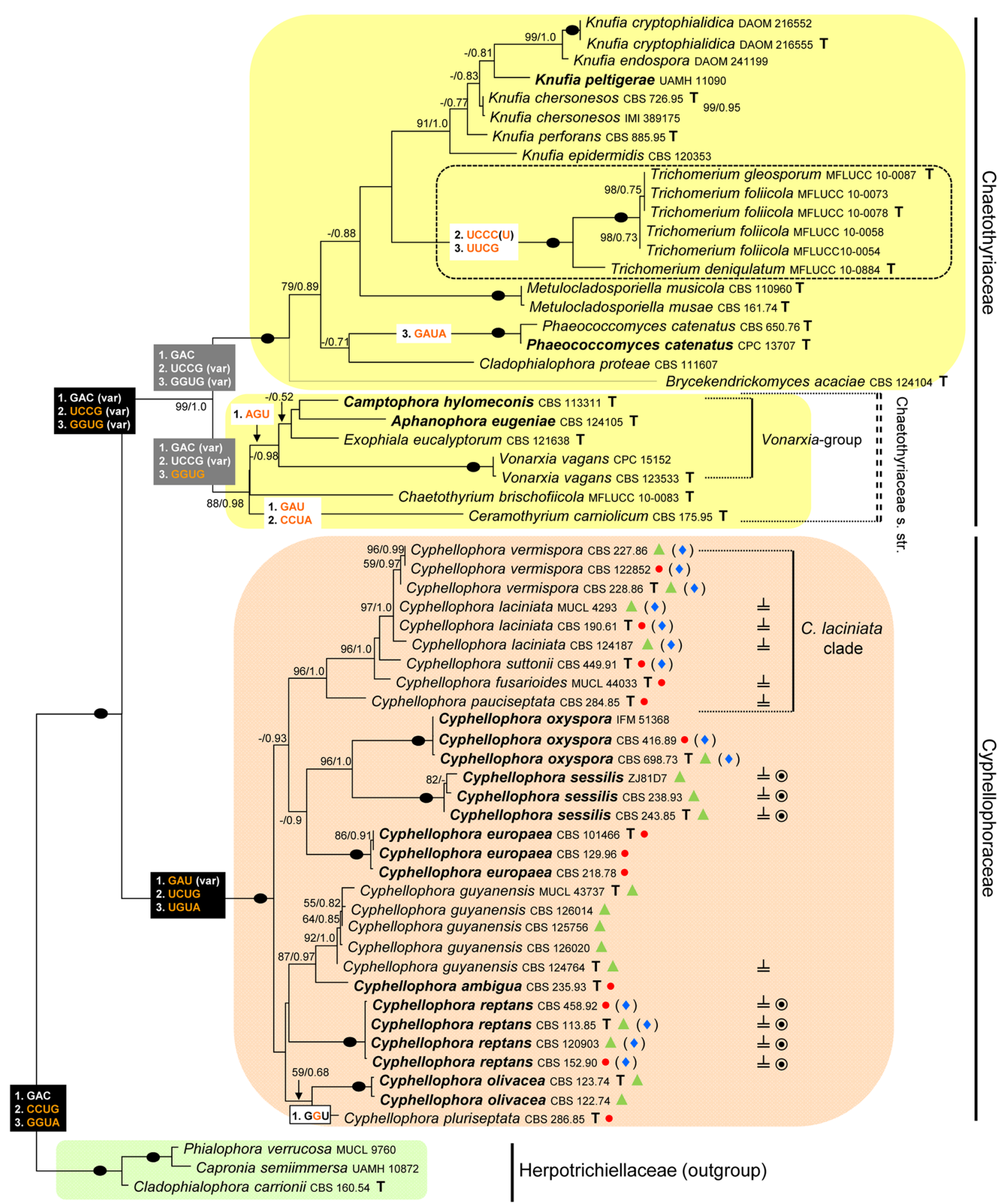


Figure 1. Phylogenetic analysis of ITS sequences of $\mathbf{3 6}$ members of the Chaetothyriales. Phylogram inferred from the ML analysis with RAXML using the GTRCAT model of evolution. Taxa in bold refer to new combinations or new taxonomic treatments. The three evolutionary motifs identified in the 2D structure of ITS2 and unique for certain phylogenetic groups are mapped on the phylogram. The abbreviation (var) indicates certain motifs deviations.

doi:10.1371/journal.pone.0063547.g001

During investigations of the systematics of Phialophora section Catenulatae W. Gams we studied the eleven species originally classified in this section [19], a number of which proved to be members of the distantly related lineages Leotiomycetes and Sordariomycetes $[20,21,22,23]$. In preliminary phylogenies based on sequences of ITS, two ribosomal DNA and three proteincoding genes, only three remaining species were found to be related to the Chaetothyriales (Eurotiomycetes), viz. Phialophora clavispora W. Gams, P. olivacea W. Gams and P. oxyspora W. Gams. The latter two species were members of a monophyletic clade that was sister to the lineage containing $P$. verrucosa Medlar, the type species of Phialophora Medlar (Herpotrichiellaceae). The clade that included $P$. olivacea and $P$. oxyspora also encompassed $P$. europaea de Hoog, Mayser \& Haase, P. reptans de Hoog and P. sessilis de Hoog, taxa referred originally to the $P$. verrucosa complex [5,24], and the recently described $P$. ambigua P. Feng \& de Hoog [11]. Cyphellophora laciniata and seven other members of the genus were also situated in the same clade.

The six Phialophora positioned as sister to the Herpotrichiellaceae are characterized by subhyaline to lightly pigmented, cylindricalelongate or occasionally flask-shaped phialides with narrowly cylindrical to funnel-shaped or slightly flaring collarettes that can be slightly darker than the lower part of the phialide. Only $P$. reptans and $P$. sessilis possess intercalary phialides with sessile collarettes and phialidic loci borne directly on undifferentiated hyphae. Conidia are subhyaline, obovoidal, clavate, ellipsoidal or fusiform, and adhere in chains or slimy heads. Among these species, only $P$. ambigua, $P$. europaea, $P$. oxyspora and $P$. reptans are involved in superficial infections of humans $[5,11,24]$. The three other Phialophora in this clade have been isolated from plants, fungi, moist wallpaper or nutrient-poor substrates such as marble, stalactites or resin $[5,19,24,25]$. All of these taxa can be differentiated from $P$. verrucosa and $P$. americana (Nannf.) S. Hughes (Herpotrichiellaceae) by their slow-growing colonies and phialides with smaller, funnel-shaped and less deeply pigmented collarettes $[5,18,24,25]$. They resemble species of Cyphellophora in possessing similar conidiogenous cells with smaller, slightly darker collarettes, but differ mainly by their shorter, nonseptate conidia.

The study described in this paper was undertaken to clarify the phylogenetic relationships of Cyphellophora and several phialophoralike species that have been resolved repeatedly as comprising a group that is sister to the Herpotrichiellaceae. We investigated relationships among the clades within the Chaetothyriales based on the combination of several approaches including the comparison of morphological and cultural characteristics and phylogenetic analyses of sequences of the ITS, nucl8S rDNA, nuc28S rDNA and three protein-coding genes, i.e., $\beta$-tubulin, DNA replication licensing factor $(\mathrm{mcm} 7)$ and second largest subunit of RNA polymerase II $(r p b 2)$. We also performed in-depth comparative analyses of ITS1 and ITS2 secondary (2D) structures of members of this order. ITS1 and ITS2 represent rapidly evolving regions of the rDNA operon and display high sequence variability. Although these loci are non-coding, they include functionally constrained positions required for the folding of their transcripts that allow their own splicing and the correct processing of the rDNA genes [26]. The ITS2 has been shown to retain a common core structure with hallmarks in its RNA transcripts that are evolutionarily constrained and universal among eukaryotes
[27,28,29]. These include four helices $(\mathrm{H} 1-\mathrm{H} 4)$, a pyrimidinepyrimidine mismatch in helix $\mathrm{H} 2$ and the occurrence of the YGGUY motif in helix H3. At the structural level, these characters have proven useful for molecular taxonomic concepts [30]. Evolutionary processes at the RNA structural level, that are responsible for preserving the RNA helix structure, i.e., the double-sided (compensatory base change, $\mathrm{CBC}$ ) and one-sided substitution (hemi-compensatory base change, hCBC), have been accepted as a basis for the CBC species concept [30,31]. This concept, which has been used to delimit biological species, is based on co-evolution of nucleotides involved in CBCs and hCBCs in two most conserved helices of the ITS2 molecule [30] and was used in our study to examine relationships among closely related Cyphellophora.

Finally, to study relationships among major clades of the order and among species of Cyphellophora and Phialophora at the structural level of the rRNA, we (1) built consensus 2D structures of ITS1 and ITS2, (2) searched for non-conserved co-evolving nucleotides that maintain base pairing in the RNA transcript, and (3) mapped all existing substitutions on to the predicted 2D models of ITS 1 and ITS2.

\section{Materials and Methods}

\section{Morphological characterization of fungal strains}

Conidiophores, conidiogenous cells and conidia were examined in water, Melzer's reagent or $90 \%$ lactic acid. Images were captured using differential interference (DIC) or phase contrast (PC) microscopy using an Olympus DP70 Camera operated by Imaging Software Cell on an Olympus BX51 compound microscope.

Strains were grown on potato-carrot agar (PCA), oatmeal agar (OA) and 2\% malt extract agar (MEA) [32] in the dark and under near-UV light source (12 h light: $12 \mathrm{~h}$ dark). Colonies were examined after 7, 21 and $30 \mathrm{~d}$ at $25^{\circ} \mathrm{C}$. Images of taxa presented in this study are of $30 \mathrm{~d}$ old cultures on PCA, if not stated otherwise. Cultures are maintained at the Centre for Agricultural Bioscience International (CABI, formerly IMI), Egham, Surrey, UK, CBS-KNAW Fungal Biodiversity Center (CBS), Utrecht, The Netherlands, the culture collection of P.W. Crous (CPG at CBS), the Canadian Collection of Fungal Cultures (DAOM), Agriculture and Agri-Food Canada, Ottawa, Canada, the Mae Fah Luang University Culture Collection, Thailand (MFLUCG), Mycothèque de l'Université catholique de Louvain (MUCL), Louvain-la-Neuve, Belgium, the University of Alberta Microfungus Collection and Herbarium (UAMH), Edmonton, Canada, and the culture collection of W.A. Untereiner (WUG) at Brandon University, Canada.

\section{DNA extraction, amplification and sequencing}

Cultures used for DNA isolations were grown as described previously $[23,33]$ and total nucleic acids were extracted from mycelia following the protocols of Lee and Taylor [34] or Réblová et al. [23]. Procedures for amplifying and sequencing the ITS, nuc18S, nuc28S and $r p b 2$ were performed as described in Bogale $e t$ al. [22] and Réblová et al. [23]. The $m \mathrm{~cm} 7$ was amplified and sequenced using primers Mcm7-709for and Mcm7-1348rev/ 
ITS- $\beta$-tubulin maximum-likelihood tree (RAxML)

39 sequences of 20 species, 1220 sites: ITS $=747, \beta$-tubulin $=473$.

Symbol indicates branch support: BS/ML and $\mathrm{BS} / \mathrm{pP}=100 \%$ or 1.0

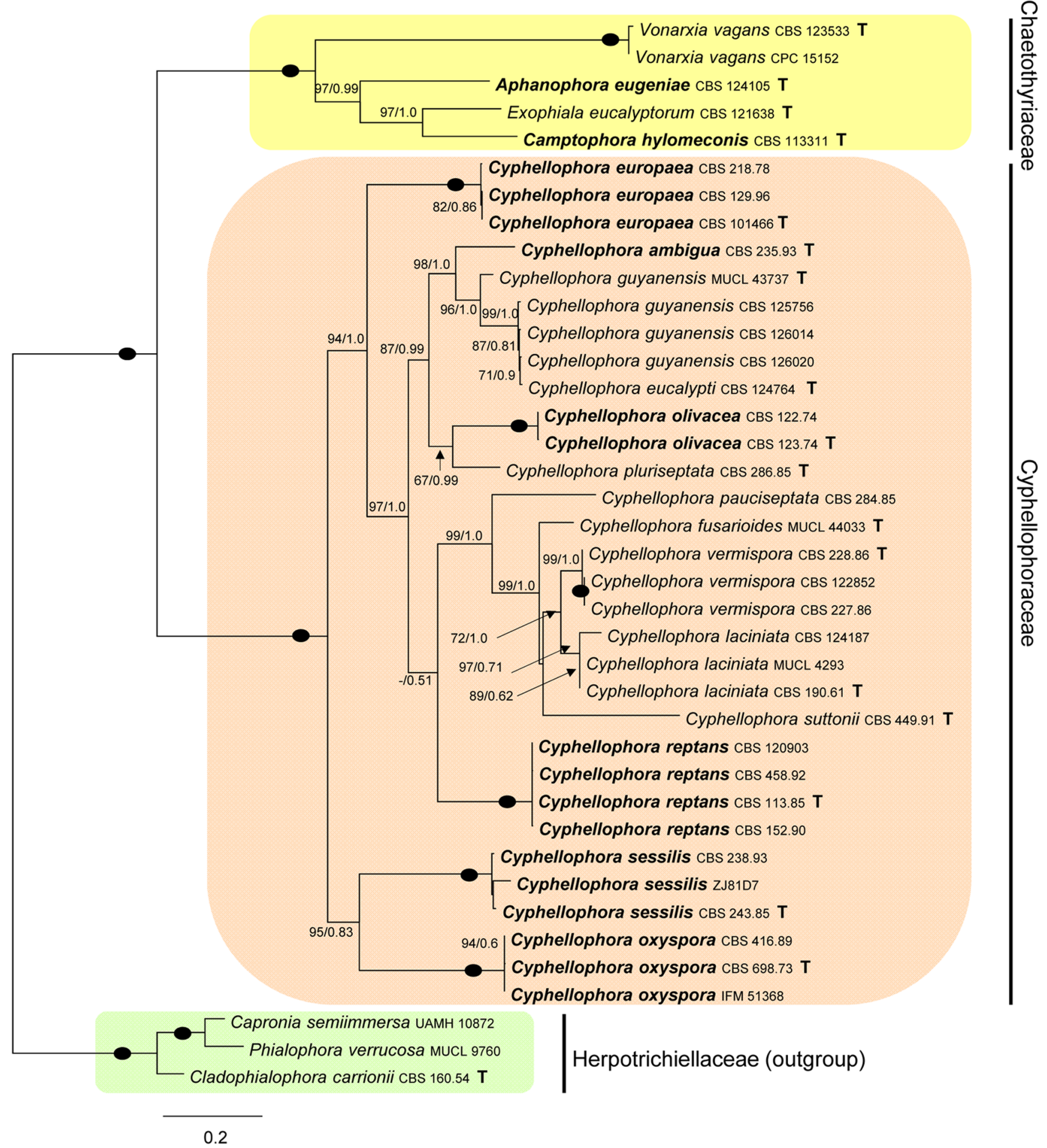

Figure 2. Phylogenetic analysis of the combined ITS rDNA and $\beta$-tubulin sequences of 20 members of the Chaetothyriales. Phylogram inferred from the ML analysis with RAxML using the GTRCAT model of evolution. Taxa in bold refer to new combinations or new taxonomic treatments.

doi:10.1371/journal.pone.0063547.g002 
four-gene maximum-likelihood tree (RAxML)

66 sequences of 45 species, 4613 sites: $\mathrm{nc} 28 \mathrm{~S}=1187, \mathrm{nc} 18 \mathrm{~S}=1668, \mathrm{rpb} 2=1128, \mathrm{mcm} 7=630$

Symbol $\bullet$ indicates branch support: BS/ML and BS/pP $=100 \%$ or 1.0

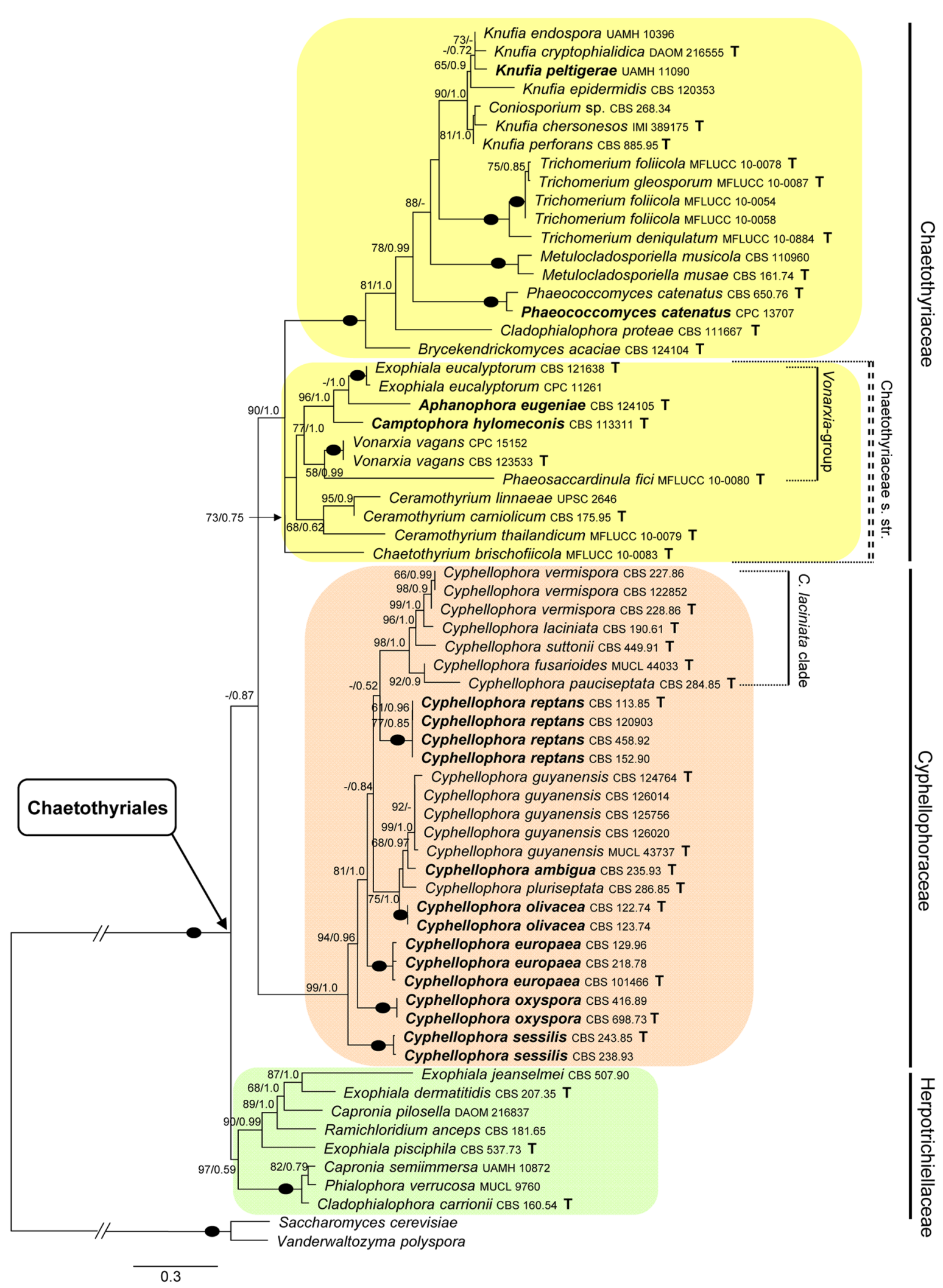


Figure 3. Multilocus phylogenetic analysis of the nuc28S-nuc18S- rpb2- $\mathrm{mcm} 7$ sequences of 45 members of the Chaetothyriales. Phylogram inferred from the ML analysis with RAXML using the GTRCAT model of evolution. Taxa in bold refer to new combinations or new taxonomic treatments.

doi:10.1371/journal.pone.0063547.g003

Mcm7-1447rev [35]. Sequences were edited using Sequencher 5.0 software (Gene Codes Corp., Ann Arbor, MI, USA).

\section{Sequence alignment}

ITS, nuc28S, nuc18S, $\beta$-tubulin, mom7 and rpb2 sequences determined for this study and homologous sequences retrieved from GeneBank are listed in Table S1. The selection of retrieved sequences was based on top hits for each gene obtained from BLAST searches. The retrieved sequences were mostly published in studies by Hoog et al. [5,24], Decock et al. [13], Crous et al. [14,17,36], Untereiner et al. [18], Tsuneda et al. [37] and Chomnunti et al. [38,39].

Sequences were manually aligned in BioEdit v.7.0.9.0 [40]. Nuc18S and nuc28S alignments were enhanced by utilizing the homologous 2D structure of Saccharomyces cerevisiae Meyen ex E.C. Hansen $[41,42]$ to improve decisions regarding homologous characters and introduction of gaps. 2D models obtained for the ITS1 and ITS2 (see below) were used to determine the positions of homologous nucleotides in the ITS. The alignment of sequences of $\beta$-tubulin, $r p b 2$ and $m c m 7$ genes were obtained as described in Réblová and Réblová [43].

Three multiple sequence alignments (MSAs) were constructed: 1) ITS, 2) ITS combined with $\beta$-tubulin, and 3) nucloS and nuc28S combined with $m c m 7$ and $r p b 2$. Individual alignments were concatenated to combine sequences for the bi- and multilocus alignments. Alignments are deposited in TreeBASE (Study no. 13812).

\section{Phylogenetic analyses}

Phylogenetic relationships were resolved based on analyses of ITS, $\beta$-tubulin, nuc18S, nuc28S, $\mathrm{mcm} 7$ and $r p b 2$ sequences of 70 isolates representing 46 species from three families of the Chaetothyriales. We analyzed the first two-thirds of the $5^{\prime}$ half of the nuc28S (corresponding to the first 1197 nucleotides of Saccharomyces cerevisiae), the almost entire nuc18S, partial $\mathrm{mcm} 7$ and the 5-7 segments of the rpb2. Bases $1-76$ of the nuclos and nuc28S alignments, $1-60$ of the $r p b 2$ and $1-78$ of the $\beta$-tubulin alignments were excluded from analyses because of the incompleteness of the $5^{\prime}$-end of the majority of the available sequences. Two outgroups were used for the three phylogenies. Members of the Herpotrichiellaceae were used to root the ITS and ITS- $\beta$ tubulin phylogenetic trees. Saccharomyces cerevisiae (nucl8S J01353, nuc28S J01355, rpb2 M15693) and Vanderwaltozyma polyspora (Van der Walt) Kurtzman (nuc18S X83825, nuc28S AY048169, rpb2 EF599484) (Saccharomycetes) were used to root the multilocus phylogeny.

Each combined data set was partitioned into several subsets of nucleotide sites. The ITS- $\beta$-tubulin analysis was partitioned into 1) ITS, and 2-4) first, second and third codon positions of $\beta$-tubulin. The four-gene data set was partitioned into 1) nuc28S, 2) nuc18S, 3-5) first, second and third codon positions of $r p b 2$, and 6-8) first, second and third codon positions of $\mathrm{mcm} 7$.

MrModeltest2 v.2.3 [44] was used to infer the appropriate substitution model that would best fit the model of DNA evolution for each sequence data set and each partition of the combined data sets. Maximum likelihood (ML) and Bayesian inference (BI) analyses were used to estimate phylogenetic relationships. ML analysis was performed with RAxML-HPG v.7.0.3 [45,46] with a GTRCAT model of evolution. Nodal support was determined by nonparametric bootstrapping (BS) with 1000 replicates. BI analysis was performed in a likelihood framework as implemented in MrBayes v.3.0b4 to reconstruct phylogenetic trees [47]. For the ITS data set we selected the GTR+G substitution model for BI analysis. For the ITS- $\beta$-tubulin data set we used a GTR+G substitution model for the first codon position of $\beta$-tubulin, a $\mathrm{HKY}+\mathrm{G}$ model for the second position and the HKY+I+G model for the third. For the combined nucl8S, nuc28S, $m c m 7$ and $r p b 2$ data set we used different models for each partition: GTR+I+G for nuc28S, nucl8S and first codon position of $m \mathrm{~cm} 7$; GTR $+\mathrm{G}$ for the first and second codon position of $r p b 2$ and second codon of $\mathrm{mcm} 7$; HKY $+\mathrm{I}+\mathrm{G}$ for the third codon position of $r p b 2$; GTR+I for the third codon position of $\mathrm{mcm} 7$. Multiple Bayesian searches using Metropolis-coupled Markov chain Monte Carlo sampling were conducted. One cold and three heated Markov chains were used in the analysis. Analyses were run for 5 million generations, with trees sampled every 1000 generations. The first 20000 trees, which represented the burn-in phase of the analysis, were discarded. The remaining trees were used for calculating posterior probabilities (PP) of recovered branches [48] in the 50\% majority rule consensus tree.

\section{Prediction of 2D structure models of ITS1 and ITS2}

Predicting the 2D structure of the ITS is essential for constructing a reliable MSA to compare nucleotides at homologous positions (in helices and loops) while searching for nonconserved co-evolving nucleotides that maintain base pairing. Consensus 2D structure models for the ITS1 and ITS2 were built using the PPfold program v.3.0 [49] which uses an explicit evolutionary model and a probabilistic model of structures, and relies on multiple sequence alignment of related RNA sequences. Final 2D models created for all members of the Chaetothyriales were further improved using Mfold program [50] and then adjusted manually if necessary, based on comparison of homologous positions in the MSA. The predicted 2D RNA structures were obtained in a dot bracket notation and were visualized and drawn using VARNA: Visualization Applet for RNA program [51]. The ITS2 Database III [52] with available structural models was also used to build consensus $2 \mathrm{D}$ structures for members of the Chaetothyriales.

\section{Identification and mapping of base-pair changes in 2D structures of ITS}

We identified three types of substitutions in the aligned ITS sequences. The first involves compensatory base changes (CBCs) that occur when both nucleotides of a paired site mutate (i.e., $\mathrm{G}=\mathrm{C} \leftrightarrow \mathrm{G}=\mathrm{G}, \mathrm{A}-\mathrm{U}$ or $\mathrm{U}-\mathrm{A})$. Because these canonical base pairs are isosterical [53] they can be substituted for each other within stems without causing structural perturbations. The second type of substitution, the hemi-compensatory base change (hCBC), entails the change of a canonical base pair to a 'wobble' base pair (i.e., $\mathrm{G}=\mathrm{C} \rightarrow \mathrm{G} / \mathrm{U}$ ). This type of substitution, which is classified as near-isosteric [54], retains base pairing in RNA molecules but produces minor structural perturbations in the helix structure because the canonical $\mathrm{G}=\mathrm{C}$ pair and wobble $\mathrm{G} / \mathrm{U}$ pair are not members of the same isosterical family [53]. Finally, we identified non-compensatory base changes (non-GBCs) that involve the replacement of a canonical pair or a wobble pair with any noncanonical pair. CBCs and hCBCs are responsible for maintaining 
A)

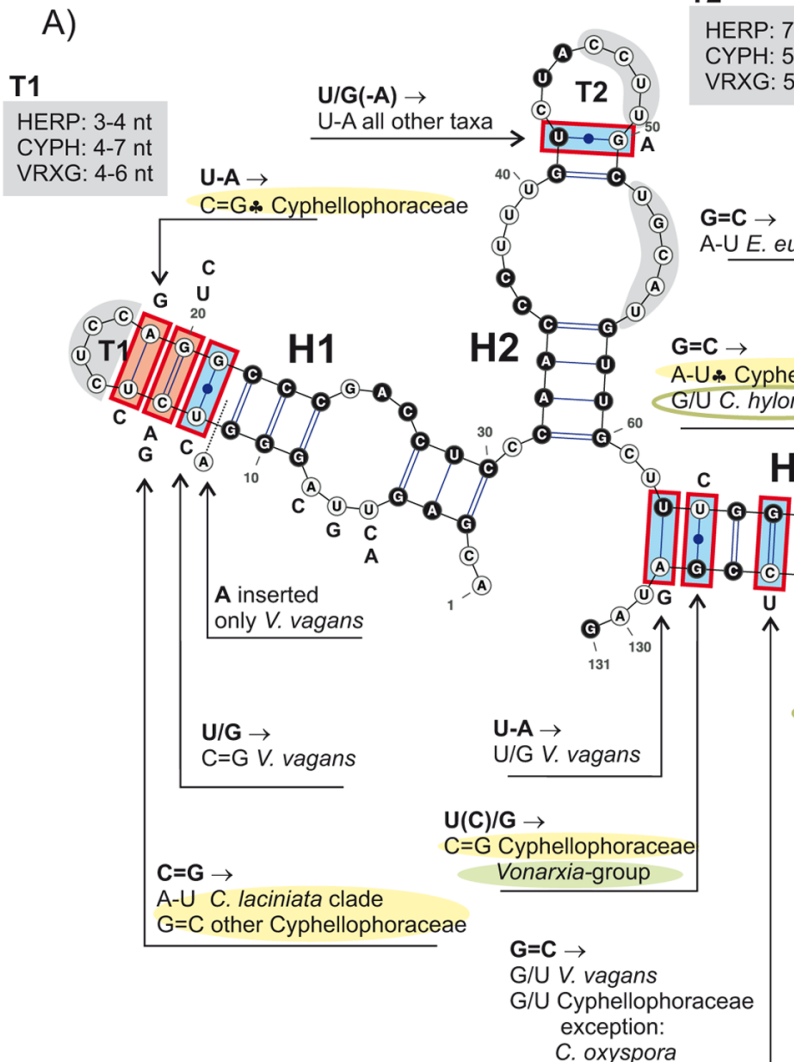

Herpotrichiellaceae

2D of ITS1 of Phialophora verrucosa

B)

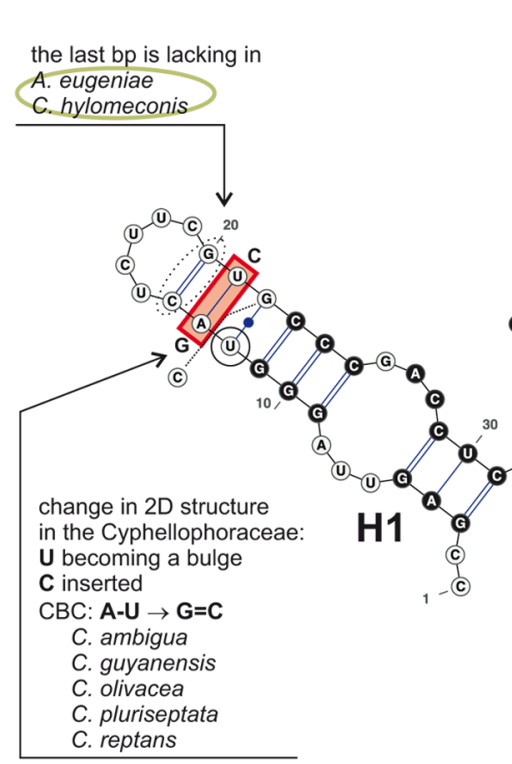

Cyphellophoraceae

2D of ITS1 of Cyphellophora laciniata

\section{C. oxyspora}

T2

RP: $7(8) \mathrm{nt}$

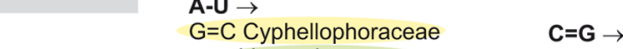

$\begin{array}{cl}\begin{array}{c}\text { Vonarxia-group } \\ \text { Vonphello }\end{array} & \mathrm{C}=\mathrm{G} \rightarrow \\ \mathrm{C} / \mathrm{A} C \text {. suttonii }\end{array}$

$\begin{array}{ll}\mathrm{U}-\mathrm{A} \rightarrow & \text { C. vermispora } \\ \text { C. pauciseptata }\end{array}$

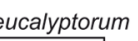

$\mathrm{C}=\mathrm{G}$ \% Cyphellophoraceae U/G C. oxyspora

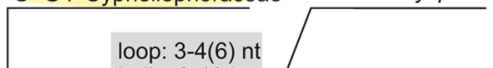

loop: $3-4(6) \mathrm{nt}$
helix: $3-12$ bp

ellophoracea

$\begin{array}{llll}\downarrow & \downarrow & \downarrow \\ A & A & G\end{array}$
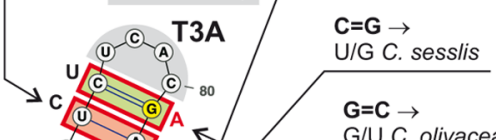

(u) $\mathrm{G}=\mathrm{C} \rightarrow$

C. pauciseptata
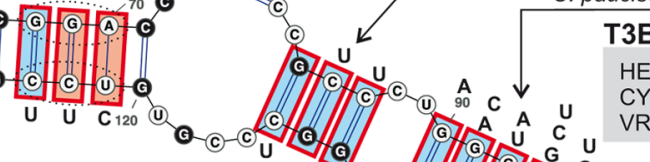

$\mathrm{G}=\mathrm{C} \rightarrow$
$\mathrm{G} / \mathrm{U}$ C. hylomeconis

C. oxyspora

C. laciniata clade

exception:

C. fusarioides $(\mathrm{G}=\mathrm{C})$

$\mathbf{C}=\mathbf{G} \rightarrow$

U/G C. europaea

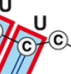

(C) ${ }_{90}^{A} \mathrm{C}$ HERP: $4-5 \mathrm{nt}$

VRXG: $8-10 \mathrm{nt}$

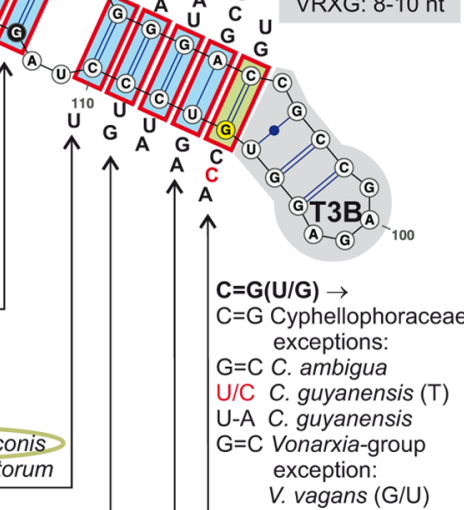

G/U C. reptans

A-U C hylomeconis E. eucalyptorum

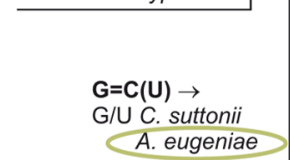

A-U $\rightarrow$

$\mathrm{G} / \mathrm{U}$ V. vagans

U/G Cyphellophoraceae

exceptions:

$\mathrm{C}=\mathrm{G}$ C. suttonii

C. guyanensis (T eucal.)

C. sessilis

U-A C. ambigua

C. pluriseptata

C. olivacea except $C$. sessilis

U-A Aphanophora, Camptophora and most members of the Chaetothyriaceae

in other taxa these two nt do not pair and become part of the loop
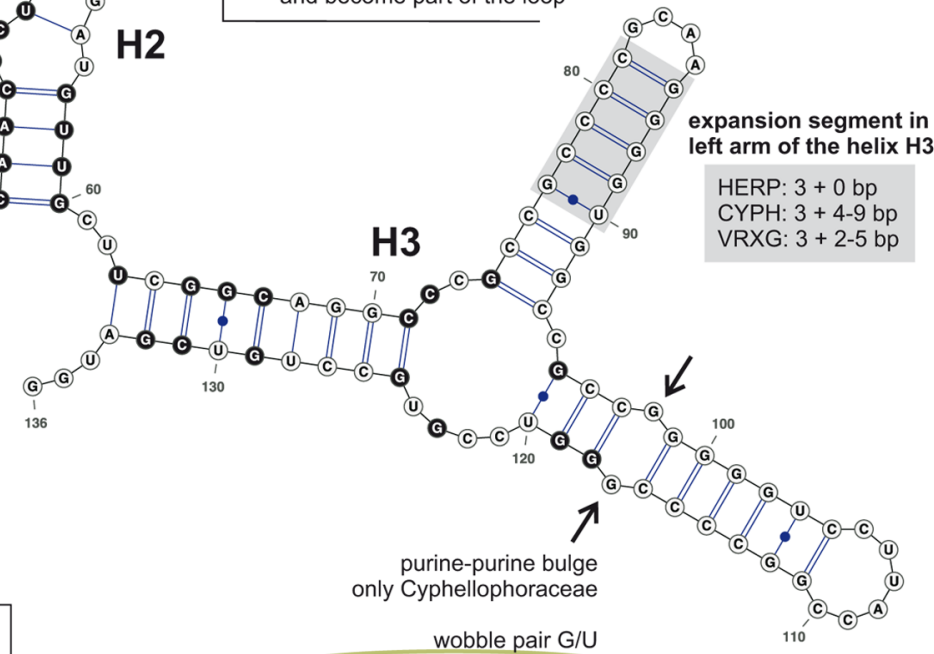
Figure 4. Partial secondary structure of ITS1 rRNA molecule showing three helices (H1-H3). A) Partial 2D structure of Phialophora verrucosa. All substitutions recorded among representatives of the Cyphellophoraceae, Herpotrichiellaceae and members of the Vonarxia-group are mapped on the 2D model. B) Partial 2D structure of Cyphellophora laciniata. Variability in the 2D structure among members of the Cyphellophoraceae and two other taxa, A. eugeniae and C. hylomeconis, segregated from Cyphellophora, is recorded on the 2D model. Abbreviations: HERP = Herpotrichiellaceae, CYPH = Cyphellophoraceae, VRXG = Vonarxia-group. Parts of hairpin loops highlighted with gray color represent regions with variable number of nucleotides. The legend to symbols and colors is included in Figure 6.

doi:10.1371/journal.pone.0063547.g004

the arrangement of base pairs in the RNA transcript and preserving the structure of the RNA helices whereas non-CBCs lead to the disruption of the stem structure [53]. Once all existing substitutions were identified among members of the Chaetothyriales, those unique to the Herpotrichiellaceae, the newly proposed family and phylogenetically related taxa of the Chaetothyriaceae s. str. were mapped onto the predicted 2D structures of ITS1 and ITS2 of Phialophora verrucosa and Cyphellophora laciniata.

\section{Nomenclature}

The electronic version of this article in Portable Document Format (PDF) in a work with an ISSN or ISBN will represent a published work according to the International Code of Nomenclature for algae, fungi, and plants, and hence the new names contained in the electronic publication of a PLOS ONE article are effectively published under that Code from the electronic edition alone, so there is no longer any need to provide printed copies.

In addition, new names contained in this work have been submitted to MycoBank from where they will be made available to the Global Names Index. The unique MycoBank number can be resolved and the associated information viewed through any standard web browser by appending the MycoBank number contained in this publication to the prefix http://www.mycobank. org $/$ MycoTaxo.aspx? Link $=\mathrm{T} \& \operatorname{Rec}=$. The online version of this work is archived and available from the following digital repositories: PubMed Central, LOCKSS.

\section{Results}

\section{Phylogenetic results}

The ITS data set for 36 species consisted of 61 sequences each with 747 characters. The ML tree is shown in Fig. 1. Three major groups were resolved: the Herpotrichiellaceae (ML BS 100\%/PP 1.0), a well-supported lineage (99/1.0) corresponding to the Chaetothyriaceae, and a robust clade (100/1.0) comprising Cyphellophora laciniata, the type species of the genus, with six other Cyphellophora and six phialophora-like taxa. This latter group represents a new family that we introduce as the Cyphellophoraceae. The core of this family is the C. laciniata subclade (96/1.0) that contains C. fusarioides (B. Sutton \& C.K. Campb.) Decock, C. laciniata, C. suttonii (Ajello, A.A. Padhye \& M. Payne) Decock, C. pauciseptata P. Feng \& de Hoog and C. vermispora A. Walz \& de Hoog. The Chaetothyriaceae encompass two subclades. The first (88/0.98) contains the Vonarxia-group (-/0.98) including Exophiala eucalyptorum Crous, Vonarxia vagans (Speg.) Aa and two Cyphellophora species, C. eugeniae Crous \& Alfenas and C. hylomeconis Crous, de Hoog \& H.D. Shin. It is sister to Ceramothyrium carniolicum (Rehm) Petr. and Chaetothyrium brischoficola (as brischofiacola) Chomnunti \& K.D. Hyde [39]. The second subclade (100/1.0) encompasses species of Cladophialophora Borelli, Knufia L.J. Hutchison \& Unter., Phaeococcomyces de Hoog, Metulocladosporiella Crous, Schroers, Groenewald, U. Braun \& K. Schubert and Trichomerium Speg. This lineage also contains Capronia peltigerae (Fuckel) D. Hawksw., which grouped with species of Knufia $(91 / 1.0)$.

The alignment for the combined ITS and $\beta$-tubulin data set consisted of 39 sequences representing 20 members of the
Chaetothyriales and 1220 characters. In the ML tree shown in Fig. 2, the Cyphellophoraceae, Herpotrichiellaceae and Chaetothyriaceae are resolved as robust clades (100/1.0). The groupings of taxa within the Cyphellophoraceae are similar to those inferred in the ITS analysis, but supports for branches in the combined ITS- $\beta$-tubulin phylogeny are generally stronger. Both $C$. eugeniae and $C$. hylomeconis are positioned again outside the Cyphellophoraceae in a clade containing members of the Chaetothyriaceae.

In the third analysis, the combined nucl8S, nuc28S, $\mathrm{mcm} 7$ and $r p b 2$ sequences were assessed for 66 taxa representing 45 species in the Chaetothyriales. This multiple alignment consisted of 4613 characters. The ML tree is shown in Fig. 3. In this phylogeny the Chaetothyriales is a strongly supported monophyletic clade (100/ 1.0) comprising three major subclades; the Cyphellophoraceae (99/1.0), Herpotrichiellaceae (97/0.59) and a large clade (90/1.0) representing the Chaetothyriaceae. As in the ITS phylogeny, the Chaetothyriaceae is divided into two lineages. The first $(73 / 0.75)$ contains three species of Ceramothyrium Bat. \& H. Maia (68/0.62), the Vonarxia-group (77/1.0), Phaeosaccardinula fici (as ficus) Chomnunti \& K.D. Hyde [38] and Chaetothyrium brischofiicola. The second subclade (100/1.0) includes the holomorph genera Knufia and Trichomerium and four genera of dematiaceous hyphomycetes. A strain of Glyphium elatum (Grev.) H. Zogg (CBS 268.34) was positioned within the genus Knufia.

\section{Consensus 2D structure of ITS1}

The 2D predicted structures of ITS1 shown in Fig. 4 are modeled for Cyphellophora laciniata and Phialophora verrucosa, representatives of the Cyphellophoraceae and Herpotrichiellaceae, respectively. The consensus 2D structure of ITS1 consisted of three helices and a variable single-stranded segment at $3^{\prime}$-end (Fig. 5A). The juxtaposed $3^{\prime}$-end folded into one or two helices that were variably positioned and separated by single-stranded regions of variable length. Because these helices are generated from non-homologous regions they cannot be compared among taxa (Fig. 5B).

The consensus 2D model of ITS1 mapped onto $P$. verrucosa (Fig. 4A) contains three helices (H1, H2 and H3) separated by an adjacent single-stranded (junction) region. $\mathrm{H} 1$ is $c$ a 30 -nucleotides (nt) long consisting of nine base pairs (bp). It exhibits a symmetrical internal loop and a hairpin loop $\mathrm{T} 1$ of a variable length. $\mathrm{H} 2$ is also ca 30-nt long but it consists of six bp. It includes an internal loop and a hairpin loop T2 that are variable in length. H3 is the longest (ca $70 \mathrm{nt}$ long) and most variable of the helices; it is also the only branched duplex in the ITS1. In the Herpotrichiellaceae it consists of a three-way junction and a short symmetrical $(2 \times 2)$ internal loop in the right arm. The latter changes to a purine-purine pair in all Cyphellophoraceae. The length of the left arm of H3 is shortest in the Herpotrichiellaceae ( $3 \mathrm{bp}$ ) but a large insertion beyond these three bp occurs in the Cyphellophoraceae and Chaetothyriaceae (Fig. 4B). The two hairpin loops T3A and T3B in $\mathrm{H} 3$ exhibit sequence variation. $\mathrm{H} 1$ and $\mathrm{H} 2$ contain six and five conserved bp in all Chaetothyriales, respectively. Other pairs in these helices are either CBCs or hCBCs. In contrast, H3 has only three conserved 
A)
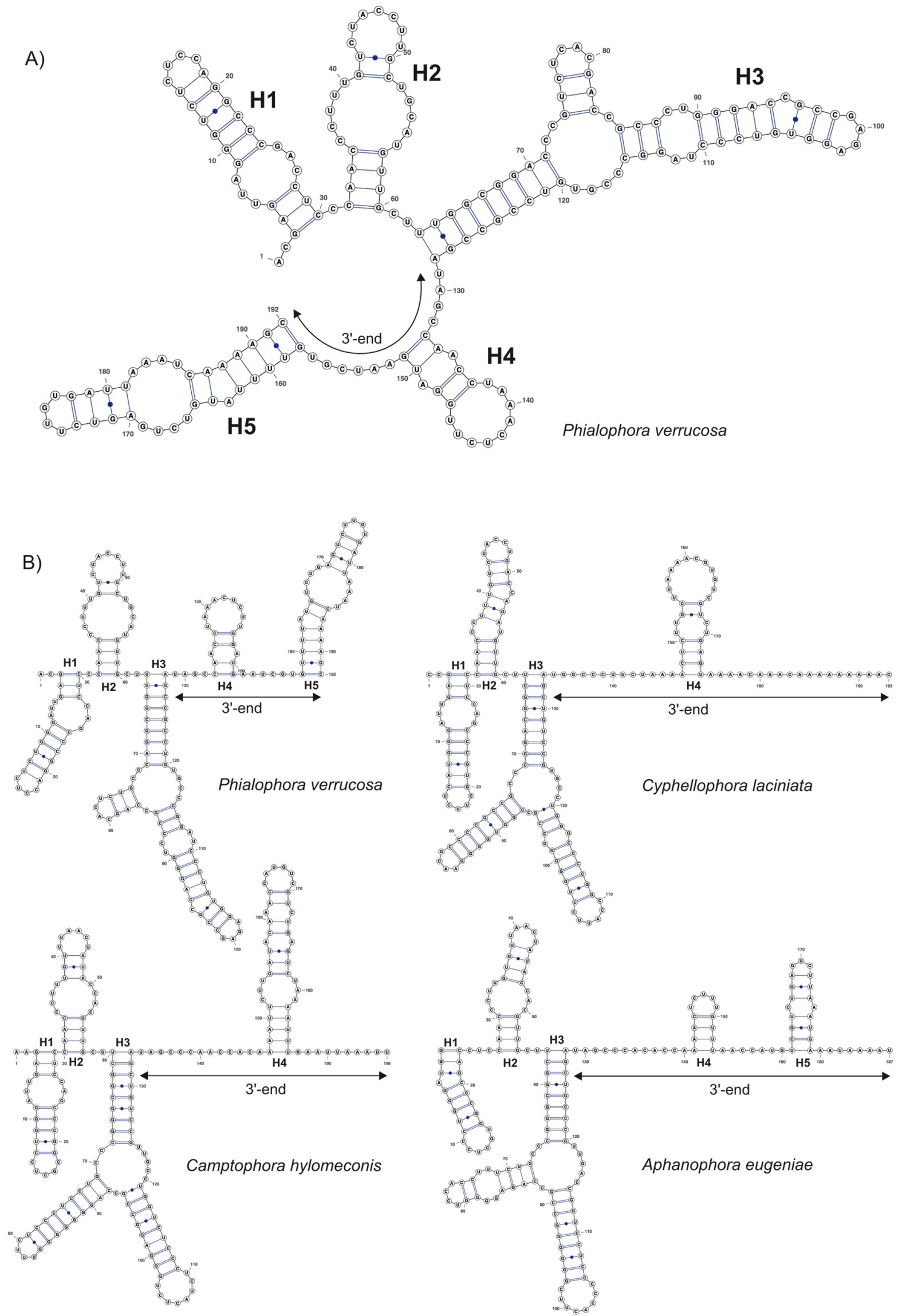
Figure 5. Predicted secondary structure models of ITS1 rRNA. A) The predicted 'ring' model of Phialophora verrucosa, the variable 3 '-end is indicated. B) The predicted 'ring' models of 2D structure of ITS1 of representative species of the Cyphellophoraceae (C. laciniata), Herpotrichiellaceae $(P$. verrucosa), and Aphanophora and Camptophora (Vonarxia-group), are transformed into linear models with helices. doi:10.1371/journal.pone.0063547.g005

bp; the two pairs in this helix are non-CBCs, others show CBCs/ hCBCs.

\section{Consensus 2D structure of ITS2}

The consensus secondary structure of ITS2 molecule mapped onto P. verrucosa (Fig. 6) and C. laciniata (Fig. 7) folded into a ring structure with four main helices $(\mathrm{H} 1-\mathrm{H} 4)$ and one alternative helix (H3A) that is inserted between $\mathrm{H} 3$ and $\mathrm{H} 4$ and separated by an adjacent single-stranded region. The ITS2 Database III [52] with available structural models was also used to build consensus 2D structures for members of the Chaetothyriales. However, the predicted structures resulted in conflicting folding patterns for individual species. In general, the 2D structures folded into four to five helices, which were generated from non-homologous sequence regions. Because such structures cannot be compared across taxa we did not use these models in our study.

$\mathrm{H} 1$ is ca 25-nt long with $8 \mathrm{bp}$, and consists of a conserved asymmetrical internal loop and a hairpin loop $\mathrm{T} 1$, which is variable. Although the primary sequence of $\mathrm{Hl}$ is constant in length among members of the Chaetothyriales, the 2D structure of the bottom portion of $\mathrm{Hl}$ is highly variable among members of the Cyphellophoraceae and the Vonarxia-group (i.e., two middle base pairs show non-CBCs that result in the disruption of the helix as illustrated for Cyphellophora europaea, C. oxyspora and Camptophora hylomeconis) (Fig. 7B).

H2 consists of ca 21-37 nt. It is composed of an asymmetrical loop and a hairpin loop $\mathrm{T} 2$, both of which exhibit a high degree of sequence variation, and a ' $\mathrm{C}$ ' bulge on the 5 ' side that is observed only in the Herpotrichiellaceae. The asymmetrical loop on the $5^{\prime}$ side consists of 4-5 nt in the Herpotrichiellaceae and 1-3 nt in other Chaetothyriales. Within the $C$. laciniata clade and in $C$. pauciseptata, this loop contains two nt, whereas other Cyphellophoraceae possess only one nt that forms a bulge. Apart from conservative bp (five in $\mathrm{H} 1$ and two in $\mathrm{H} 2$ ), both $\mathrm{H} 1$ and $\mathrm{H} 2$ contain two non-CBCs. In addition, the $\mathrm{H} 2$ helix contains several hCBCs and one $\mathrm{CBC}$.

H3 is the longest duplex. It comprises ca 73 nt and consists of up to five internal loops, one bulge and a hairpin loop T3. The shortest internal loop $(1 \times 1)$ is formed by a conserved $\mathrm{A} / \mathrm{A}$ pair. Two internal loops are asymmetrical and two others are variable in sequences among the organisms compared. In general, it is difficult to say whether they are symmetrical or asymmetrical. This helix contains nine conserved bp, two bp with CBCs, and three bp with both $\mathrm{CBC}$ s and hCBCs. Other pairs are non-CBCs. The most conserved area in the ITS2 is located near the $5^{\prime}$-end of H3. Nucleotides that are constant in all members of the Chaetothyriales are found on both sides of the upper part of H3. The fourth duplex H4 is short having one conserved bp and two bp with both CBCs and hCBCs. Its hairpin loop T4 is of variable length.

The alternative duplex $\mathrm{H} 3 \mathrm{~A}$ is positioned between $\mathrm{H} 3$ and $\mathrm{H} 4$. It is present only in the Herpotrichiellaceae, Cyphellophoraceae, and selected Chaetothyriaceae, i.e., $V$. vagans, Ceramothyrium thailandicum Chomnunti \& K.D. Hyde and Phaeococcomyces catenatus (de Hoog \& Herm.-Nijh.) de Hoog.

The 2D model of Brycekendrickomyces acaciae Crous \& M.J. Wingf. differs from those produced for the Herpotrichiellaceae, Chaetothyriaceae and Cyphellophoraceae. In this species the H3A is short and the remaining nucleotides form an adjacent singlestranded region and do not fold into H4. Two species of
Metulocladosporiella possess the longest $\mathrm{H} 4$ helix and the adjacent single-stranded region following $\mathrm{H} 3$ is missing in these taxa. All nucleotides past the conserved $\mathrm{GU}(\mathrm{C}) \mathrm{C}$ triplet (nt 151-153 of Phialophora verrucosa, Fig. 6) form a 12 bp long helix $\mathrm{H} 4$ consisting of one symmetrical and one asymmetrical internal loop.

We identified three evolutionary motifs in the 2D structure of ITS2 that are unique for certain phylogenetic groups (Table 1 and Figs. 1, 6, 7). Motif M1 (GAC, with a deviation in Ceramothyrium carniolicum as GAU) is repeated in all members of the Chaetothyriales with exception of the Vonarxia-group (AGU), and the Cyphellophoraceae $(\mathrm{GA}(\mathrm{G}) \mathrm{U})$, and is positioned in a singlestranded region between $\mathrm{H} 2$ and H3. Motifs M2 and M3 occur on the 5 'side of $\mathrm{H} 3$ (Figs. 6, 7). M2 is positioned in the lower half of the $\mathrm{H} 3$; this motif is unique for the Cyphellophoraceae (UCUG) and the Herpotrichiellaceae (CGUG), but varies within the Chaetothyriaceae (UCCG), i.e., GCUA in Ceramothyrium carniolicum, UCAG in Cladophialophora proteae Viljoen \& Crous and $\mathrm{UCCC}(\mathrm{U})$ in Trichomerium. M3 is situated in the lower portion of the highly conserved region near the hairpin loop T3 and defines the Cyphellophoraceae (UGUA), Herpotrichiellaceae (GGUA) and some Chaetothyriaceae (GGUG).

\section{Taxonomy}

Cyphellophoraceae. Réblová \& Unter., fam. nov.

[urn:lsid:indexfungorum.org:names: 803682]

Mycelium composed of hyaline or pigmented hyphae. Conidiophores mostly formed as simple phialides. Phialides discrete, intercalary, terminal or lateral, sometimes very short, flask-shaped, elongate flask-shaped, or cylindrical elongate and then hardly tapering towards the collarette. Collarettes funnel-shaped with flaring opening or cylindrical and hardly divergent, hyaline or slightly darker than the lower part of the phialide. Conidia nonseptate or septate, hyaline, subhyaline or pale brown, ranging from obovoidal or ellipsoidal to fusiform, cylindrical, acicular, straight, curved or sigmoid. Budding and germinating cells present. Teleomorphs unknown.

Type genus. Cyphellophora G.A. de Vries emend. Réblová \& Unter.

Cyphellophora. G.A. de Vries emend. Réblová \& Unter.

[urn:lsid:indexfungorum.org:names:7885]

= Kumbhamaya M. Jacob \& D. J. Bhat, Cryptog. Mycol. 21: 82. 2000.

Mycelium composed of branched, septate, hyaline or slightly pigmented hyphae. Conidiophores mostly formed as simple phialides, rarely with an additional septum. Phialides discrete, intercalary, terminal or lateral, sometimes very short, flask-shaped, elongate flask-shaped, or cylindrical elongate, hardly tapering towards the collarette. Collarettes indistinct, funnel-shaped and flaring at the opening or narrowly funnel-shaped to cylindrical and hardly divergent, hyaline or slightly darker than the lower part of the phialide. Conidia nonseptate or septate, hyaline, subhyaline or pale brown, ranging from obovoidal to ellipsoidal, clavate, oblongovoid, fusiform, cylindrical, vermiform or acicular, straight, curved, falcate or sigmoid, frequently anastomosing. Budding and germinating cells present. Teleomorphs unknown.

Type species. Cyphellophora laciniata G.A. de Vries, Mycopath. Mycol. appl. 16: 47. 1962.

Comments. The monophyletic and strongly supported clade encompassing $C$. laciniata and eight other members of the genus 


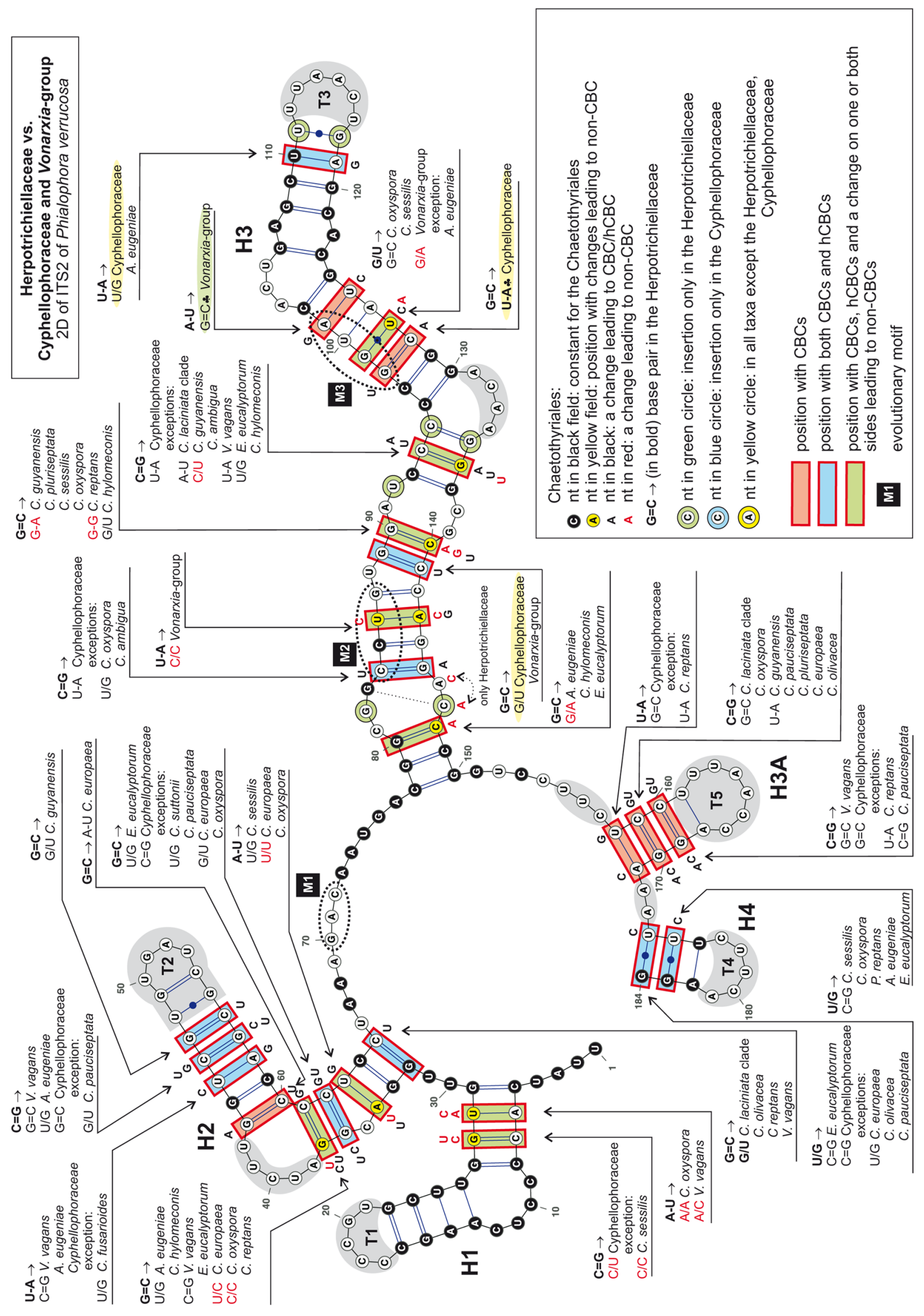


Figure 6. Predicted secondary structure model of the ITS2 rRNA molecule of Phialophora verrucosa. Five helices commonly found in the 2D structure in members of the Cyphellophoraceae and Herpotrichiellaceae are numbered $\mathrm{H} 1-\mathrm{H} 3, \mathrm{H} 3 \mathrm{~A}, \mathrm{H} 4$. All substitutions recorded among representatives of these two families and members of the Vonarxia-group are mapped on the 2D model. Parts of hairpin loops highlighted with gray color represent regions with variable number of nucleotides.

doi:10.1371/journal.pone.0063547.g006

also includes six species formerly treated as members of the genus Phialophora (Figs. 1-3). Subclades corresponding to individual species are not defined by ecology or by similarities in the morphologies of the conidia (septation, shape, presence of budding cells), collarettes (pigmentation, shape), and conidiogenous cells (shape, intercalary with sessile collarettes vs. prominent terminal and/or lateral). Because these subclades are ecologically and morphologically heterogeneous and cannot be recognized as separate genera, we accept all members of the Cyphellophoraceae clade as belonging to Cyphellophora and emend the genus to include species with nonseptate conidia and mostly cylindrical-elongate to flask-shaped elongate phialides. Accordingly, we propose six new combinations in Cyphellophora and provide a key to its species.

Cyphellophora ambigua. (P. Feng \& de Hoog) Réblová \& Unter., comb. nov.

[urn:lsid:indexfungorum.org:names: 803697]

Basionym. Phialophora ambigua P. Feng \& de Hoog, Fung. Diver. (p. 22 of the still unpaginated paper), 2012. DOI 10.1007/s13225012-0194-5.

Habitat. Isolated from a human toenail.

Illustrations. The species is illustrated in Feng et al. [11].

Cyphellophora europaea. (de Hoog, Mayser \& Haase)

Réblová \& Unter., comb. nov. (Fig. 8A-G).

[urn:lsid:indexfungorum.org:names: 803683]

Basionym. Phialophora europaea de Hoog, Mayser \& Haase, Mycoses 43: 414. 2000.

Habitat. This species has been isolated most frequently from nails and skin scrapings $[5,24]$.

Living strains examined. CBS 101466 (ex-type), CBS 129.96.

Cyphellophora olivacea. (W. Gams) Réblová \& Unter., comb. nov. (Fig. $8 \mathrm{H}-\mathrm{O}$ )

[urn:lsid:indexfungorum.org:names: 803684]

Basionym. Phialophora olivacea W. Gams, Stud. Mycol. 13: 65. 1976.

Habitat. Known from various substrates including wallpaper, stalactites, dead plants, and the fruit body of a basidiomycete [19].

Living strains examined. CBS 122.74 (ex-type), CBS 123.74.

Cyphellophora oxyspora. (W. Gams) Réblová \& Unter., comb. nov. (Fig. 9A-E)

[urn:lsid:indexfungorum.org:names: 803685]

Basionym. Phialophora olivacea W. Gams, Stud. Mycol. 13: 64. 1976.

Habitat. The ex-type strain was isolated from decaying leaves of Clerodendron monahassa (Verbenaceae) [19]. A second strain was isolated from a human toenail [11].

Living strain examined. CBS 698.73 (ex-type).

Cyphellophora reptans. (de Hoog) Réblová \& Unter., comb. nov. (Fig. 10A-K)

[urn:lsid:indexfungorum.org:names: 803686]

Basionym. Phialophora reptans de Hoog, Stud. Mycol. 43: 117. 1999.

Habitat. The ex-type strain was isolated from food. Additional strains have been isolated from human nails and skin scrapings, bark, soil, and water [24].

Living strains examined. CBS 113.85 (ex-type), CBS 458.92.

Cyphellophora sessilis. (de Hoog) Réblová \& Unter., comb. nov. (Fig. 10L-R)

[urn:Isid:indexfungorum.org:names: 803687]
Basionym. Phialophora sessilis de Hoog, Stud. Mycol. 43: 120. 1999.

Habitat. This species has been isolated from a variety of nutrient-poor, recalcitrant substrates including the resin of Picea abies (ex-type strain), styrene, and marble powder, as well as from decaying plants $[24,25]$. Phialophora sessilis has been reported as the agent of flyspeck and sooty blotch disease $[55,56]$. This species has never been documented as a cause of infections of warm- or coldblooded animals.

Living strains examined. CBS 243.85 (ex-type), CBS 238.93.

Comments. Although Cyphellophora was originally distinguished from Phialophora by its septate conidia and intercalary conidiogenous cells that did not match the concept of a phialide (i.e., a discrete cell borne at the end of a short branch or laterally) [6], the concept of the genus was later broadened to include species with discrete, terminal and lateral conidiogenous cells [9]. Key to species accepted in Cyphellophora is shown in Fig. 11.

Cyphellophora comprises the type of Kumbhamaya M. Jacob \& Bhat. Type now recognized as Cyphellophora indica (M. Jacob \& Bhat) C. Decock, a species that resembles C. pluriseptata G.A. de Vries, Elders \& Luykx in producing pale brown, flask-shaped phialides with elongated hyphal bases and septate, fusiform conidia. Cyphellophora can also be compared to Pseudomicrodochium B. Sutton. Two species originally classified in this genus, $C$. fusarioides (B. Sutton \& C.K. Campb.) Decock and C. suttonii, were transferred to Cyphellophora by Decock et al. [13] based on the comparison of cultural characters and the analysis of nuclos rDNA sequences. Pseudomicrodochium aciculare B. Sutton, the type species of this genus, has been collected frequently on the rotting cupules of Castanea sativa [57] and differs from species now treated as members of the genus Cyphellophora by the formation of white to pale salmon colonies, hyaline conidiogenous cells aggregating in sporodochia, and hyaline conidia in vitro [13]. The strain MUCL 39134 of $P$. aciculare was collected on decaying leaves and resolved as belonging to the Hypocreales [13].

The shape of the conidia of Cyphellophora ranges from short to elongate. Nonseptate conidia are obovoidal to ellipsoidal to clavate or slightly fusiform and are often truncate at the basal end, while septate conidia range from oblong-ovoid or oblong-fusoid to cylindrical to acicular to vermiform, and can be straight, curved or sigmoid. The C. laciniata clade, which is the largest group inferred in all three phylogenies (Figs. 1-3), encompasses four species with septate conidia, viz. C. fusarioides, C. laciniata, C. suttonii, and $C$. vermispora. Three other species of Cyphellophora with septate, fusiform or vermiform conidia, viz. C. pauciseptata, C. guyanensis Decock \& G. Delgado, and C. pluriseptata, are dispersed among species with nonseptate, oblong, ellipsoidal to clavate conidia. Taxa with intercalary phialides are also not restricted to specific subclades (Fig. 1). Two species with septate conidia formed from intercalary phialides are placed in the $C$. laciniata clade $(C$. laciniata, C. fusarioides). A third species sharing these features, C. pauciseptata, is sister to the $C$. laciniata clade, while $C$. guyanensis, which produces septate conidia but seldom forms intercalary phialides, is positioned outside the core group. Cyphellophora reptans and $C$. sessilis are the only members of the genus with nonseptate conidia and intercalary phialides. These taxa exhibit yet another morphological peculiarity; in axenic culture they produce germinating (C. reptans in Fig. 10C, G) and budding cells (C. reptans in 


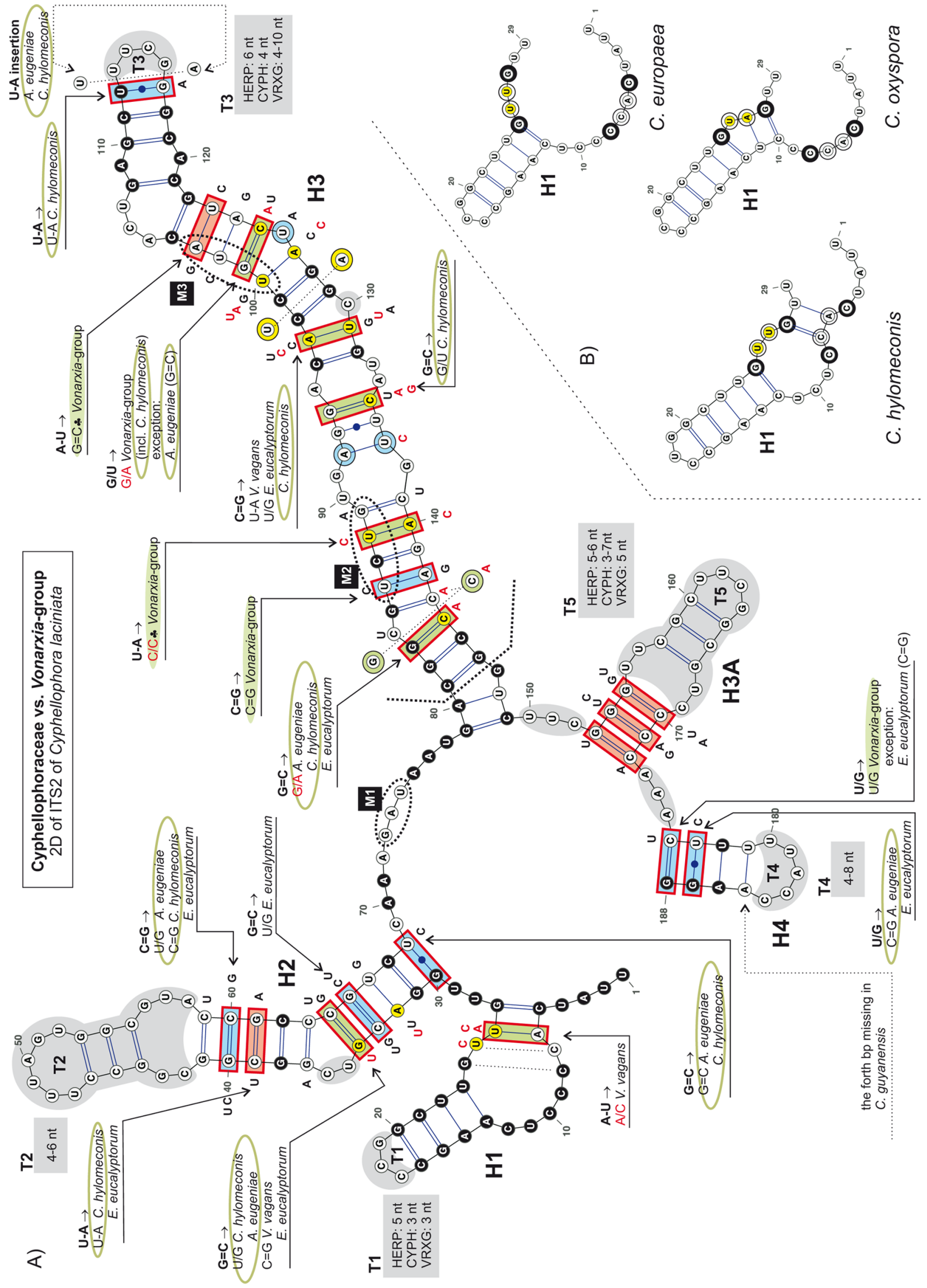


Figure 7. Predicted secondary structure model of the ITS2 rRNA molecule of Cyphellophora laciniata. Substitutions that characterize Cyphellophoraceae and members of the Vonarxia-group are mapped on the 2D model. Parts of hairpin loops highlighted with gray color represent regions with variable number of nucleotides. The legend to symbols and colors is included in Figure 6. doi:10.1371/journal.pone.0063547.g007

Fig. 10I, J and C. sessilis in Fig. 10M, N) and form yeast-like colonies (Fig. 10A, L) that are reminiscent of some Exophiala J.W. Carmich. (Herpotrichiellaceae) [33,58]. Other Cyphellophoraceae produce slow growing, dark colonies in vitro with abundant aerial mycelium and lack budding cells. The term budding cells was first used by de Hoog et al. [58], in fact these cells are swollen conidia with a phialidic opening.

Information on the ecology and potential pathogenicity of the Cyphellophoraceae is incomplete. Teleomorphs are unknown for this family and all Cyphellophora species, except $C$. europaea, $C$. laciniata, C. reptans and C. sessilis, are known from three or fewer isolates. Cyphellophora laciniata [6], C. europaea [5], C. oxyspora [11], C. pauciseptata [11], C. pluriseptata [9], C. reptans [24], C. suttonii [59], and $C$. vermispora [11] have been isolated from human samples. Cyphellophora suttonii is also reported from animal skin (subcutaneous ear lesion) [8] and C. fusarioides has been isolated from pulmonary fluids [10]. Among these species, only C. fusarioides, $C$. pauciseptata, and C. pluriseptata are known solely from clinical isolates. The other species are also known from nonclinical sources. For example, strains of $C$. europaea were reported from environmental samples from humid environments (i.e., bathrooms, washing machines) [60,61], C. reptans was originally isolated from food [24], a strain of $C$. laciniata was isolated from a bathroom surface, and the ex-type strain of $C$. oxyspora was isolated from decaying leaf [19]. Cyphellophora suttonii was also detected in soil samples, although this strain has never been sequenced [12]. Other Cyphellophora that have not been involved in human infections are frequently isolated from plants or nutrient-poor substrates, and two species (C. olivacea and C. oxyspora) were originally classified in the Phialophora sect. Catenulatae [19].

Although several Cyphellophora have been isolated from clinical sources, these fungi are not necessarily agents of infection. Nondermatophytic filamentous fungi (NDF) (i.e., species other than Arthrodermataceae) rarely infect the nails and skin of humans and animals, but it is difficult to compare the accuracy of various procedures used to determine the agents of these infections. The current standard procedure for confirming NDF

Table 1. A list of evolutionary motifs $(M 1-M 3)$ recorded in the ITS2 molecule of members of the Chaetothyriales.

\begin{tabular}{llll}
\hline Taxa and families & M1 & M2 & M3 \\
\hline Herpotrichiellaceae & GAC & CCUG & GGUA \\
Cyphellophoraceae & GAU $^{\text {a }}$ & UCUG & UGUA \\
Chaetothyriaceae & GAC $^{*}$ & UCCG* & GGUG* \\
Ceramothyrium carniolicum & $\mathrm{GAU}^{*}$ & CCUA $^{*}$ & GGUG \\
Cladophialophora proteae & GAC & UCAG* $^{*}$ & GGUG \\
Phaeococcomyces catenatus & GAC & UCCG & GAUA* \\
Trichomerium & GAC & UCCC ${ }^{*}$ & UUCG* \\
Vonarxia-group & AGU* & UCCG & GGUG \\
\hline
\end{tabular}

Motifs in bold determine a group at family level. Taxa or groups of taxa that belong to the Chaetothyriaceae and show certain motifs deviations are listed. *Asterisk indicates presence of more than one unique motif within the group. (a,b) deviations: ${ }^{\mathrm{a}} \mathrm{GGU}$ in Cyphellophora pluriseptata, ${ }^{\mathrm{b}} \mathrm{UCCU}$ in Trichomerium deniqulatum.

doi:10.1371/journal.pone.0063547.t001 as specialized for pathogenesis of nails (onychomycosis) recommends that the infecting fungus be isolated from at least two successive but temporally separate nail specimens, at least one of which is consistent for compatible filaments observed in direct $\mathrm{KOH}$ microscopy [62]. This is because nails with medical problems have fissures and rough surfaces that trap or contain fungi, dust and dirt which could result in the misinterpretation of the etiology of a species from a single isolation [62] ( $R$. Summerbell pers. com.). We therefore advocate the careful evaluation of the etiology of both dermatomycoses and onychomycoses attributed to the Cyphellophoraceae before characterizing these fungi as capable of infecting nails and skin.

Aphanophora. Réblová \& Unter., gen. nov.

[urn:lsid:indexfungorum.org:names: 803677]

Mycelium immersed and superficial, composed of branched, septate, pigmented hyphae. Conidiophores absent, reduced to a single conidiogenous cell. Setae present. Conidiogenous cells phialidic, intercalary, seldom terminal, multiple conidiogenous loci within the collarette; collarettes inconspicuous, hyaline. Conidia hyaline to subhyaline, composed of subcylindrical to cylindrical segments, marked by constrictions in the wall at the septa. Each segment divided by a secondary median septum. Conidia anastomose and undergo microcyclic conidiation in culture.

Etymology. Aphanos $(\mathrm{Gk})=$ inconspicuous, referring to the inconspicuous multiple conidiogenous loci within the collarette; phora $(\mathrm{Gk})=$ bearing from pherein $=$ to bear.

Type species. Aphanophora eugeniae (Crous, de Hoog \& H.D. Shin) Réblová \& Unter.

Aphanophora eugeniae. (Crous \& Alfenas) Réblová \& Unter., comb. nov. (Fig. 12A-I)

[urn:lsid:indexfungorum.org:names: 803678]

Basionym. Cyphellophora eugeniae Crous \& Alfenas, Persoonia 22: 147. 2009.

Habitat. Isolated from living leaves of Eugenia uniflora (= Stenocalyx uniflorus) (Myrtaceae) [17].

Living strain examined. CBS 124105 (ex-type).

Camptophora. Réblová \& Unter., gen. nov.

[urn:lsid:indexfungorum.org:names: 803679]

Mycelium immersed and superficial, composed of branched, septate, pigmented hyphae. Conidiophores absent, reduced to a single conidiogenous cell. Conidiogenous cells phialidic, intercalary, seldom terminal, proliferating percurrently, single conidiogenous locus within the collarette; collarettes distinct, hyaline, narrowly funnel-shaped, hardly divergent. Conidia hyaline, subhyaline to light brown, fusiform or sickle-shaped, septate, constricted at the septa. Conidia anastomose and undergo microcyclic conidiation in culture.

Etymology. Kamptós $(\mathrm{Gk})=$ curved, referring to the curved conidia; phora $(\mathrm{Gk})=$ bearing from pherein $=$ to bear.

Type species. Camptophora hylomeconis (Crous, de Hoog \& H.D. Shin) Réblová \& Unter.

Camptophora hylomeconis. (Crous, de Hoog \& H.D. Shin) Réblová \& Unter., comb. nov. (Fig. 13A-L)

[urn:lsid:indexfungorum.org:names: 803680]

Basionym. Cyphellophora hylomeconis Crous, de Hoog \& H.D. Shin, Stud. Mycol. 58: 200. 2007.

Habitat. Isolated from living leaves of Hylomecon vernalis (Ranunculaceae) [14].

Living strain examined. CBS 113311 (ex-type). 

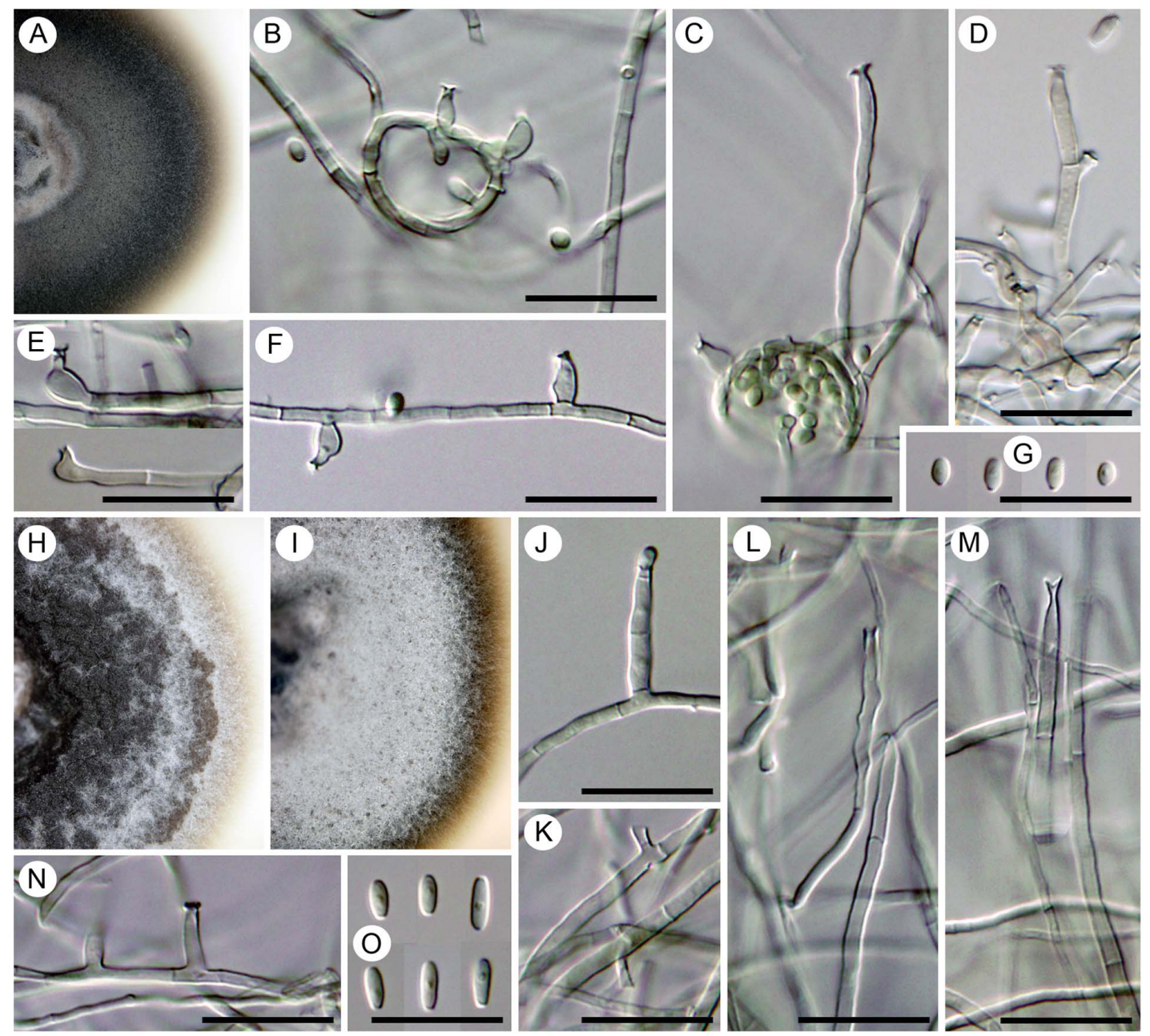

Figure 8. Two species of Cyphellophora with nonseptate conidia in vitro. A-G) Cyphellophora europaea: living culture (A), terminal or lateral phialides (B-F), and conidia (G). DIC, bar $=20 \mu \mathrm{m}$. CBS 101466 (ex-type). H-O) Cyphellophora olivacea: living culture with a detail of aerial mycelium $(\mathrm{H}, \mathrm{I})$, terminal and lateral phialides $(\mathrm{J}, \mathrm{L}-\mathrm{N})$, intercalary phialides (K), and conidia (O). DIC, bar $=20 \mu \mathrm{m}$. CBS 122.74 (ex-type), CBS 123.74 (only O). doi:10.1371/journal.pone.0063547.g008
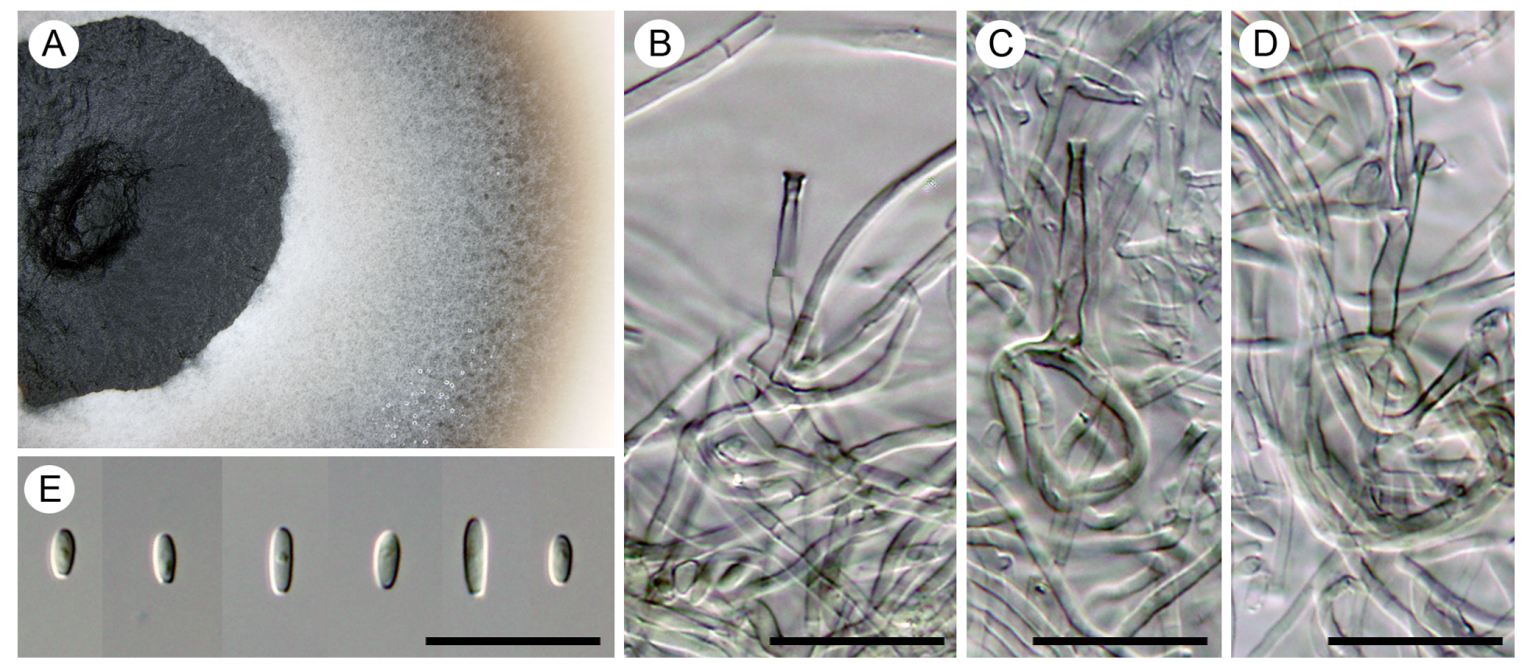

Figure 9. Species Cyphellophora oxyspora with nonseptate conidia in vitro. Living culture (A), phialides (B-D), and conidia (E). DIC, bar $=20 \mu \mathrm{m}$. CBS 698.73 (ex-type).

doi:10.1371/journal.pone.0063547.g009 

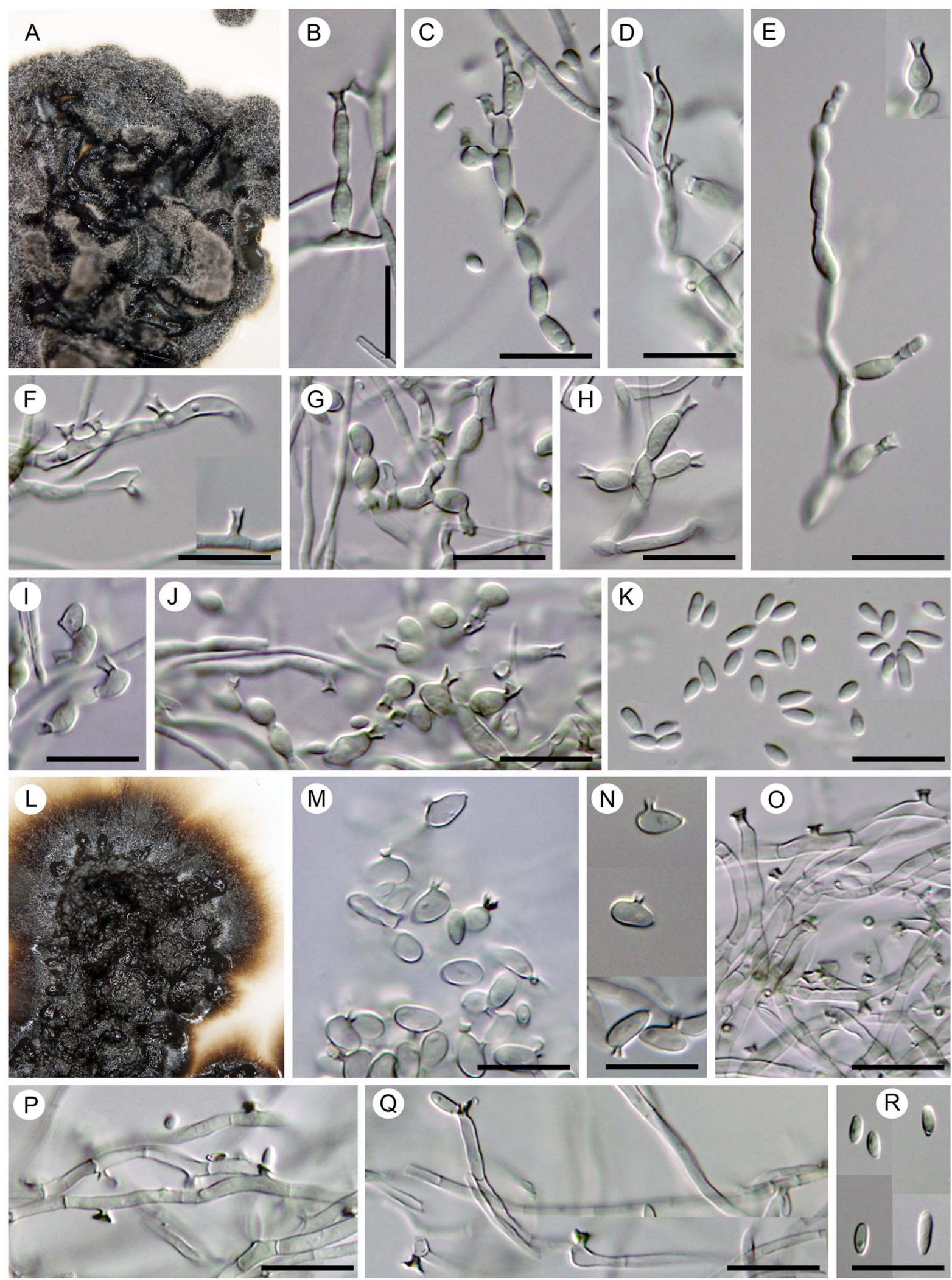

Figure 10. Two species of Cyphellophora characterized by the presence of budding and germinating cells. A-K) Cyphellophora reptans: living culture with a yeast-like appearance (A), lateral and terminal phialides (B, D, H), intercalary phialides (F), germinating cells $(C, G, I)$, budding cells $(\mathrm{J})$, and nonseptate conidia (K). DIC, bar $=20 \mu \mathrm{m}$. CBS 113.85 (ex-type, only A), CBS 458.92. L-R) Cyphellophora sessilis: Living culture with sparse aerial mycelium and a yeast-like appearance $(L)$, budding cells $(M, N)$, terminal and lateral phialides $(\mathrm{O}, \mathrm{Q})$, intercalary phialides $(\mathrm{P})$, and conidia $(\mathrm{R})$. DIC, bar $=20 \mu \mathrm{m}$. CBS 243.85 (ex-type).

doi:10.1371/journal.pone.0063547.g010 


\section{Key to species accepted in Cyphellophora}

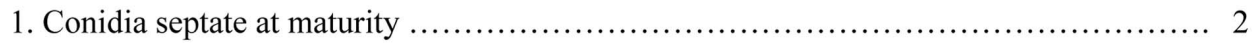

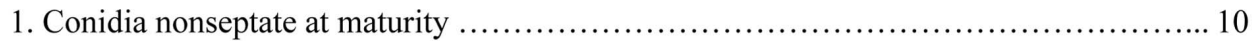

2. Phialides predominantly intercalary with a sessile, flared collarettes ............. 3

2. Phialides prominent and rising above the undifferentiated hypha, rarely intercalary, lateral or terminal, flask-shaped, cylindrical $\ldots \ldots \ldots \ldots \ldots \ldots \ldots \ldots \ldots \ldots \ldots \ldots \ldots 6$

3. Conidia up to $2.5 \mu \mathrm{m}$ wide, fusiform to falcate .................................. 4

3. Conidia up to $5 \mu \mathrm{m}$ wide $(11-25 \times 2.0-5.0 \mu \mathrm{m})$, oblong-fusoid to sickle-shaped, 1-3septate, hyaline or becoming pale brown

C. laciniata

4. Conidia up to $1.0 \mu \mathrm{m}$ wide, $(12-) 14-16(-20) \times 0.8-1.0 \mu \mathrm{m} \ldots \ldots \ldots$. . C. pauciseptata

4. Conidia wider than $1.0 \mu \mathrm{m}$

C. fusarioides

5. Conidia 11-20 × 2.0-2.5 $\mu \mathrm{m}, 1(-2)$-septate, hyaline

5. Conidia $16-35 \times 1.5-2.0 \mu \mathrm{m}, 3$-septate, pale brown ..................... C. taiwanensis
6. Phialides rarely intercalary, terminal or lateral, collarettes darker than the rest of the phialide, funnel-shaped; conidia hyaline to pale-brown, at first clavate and 1-septate becoming sigmoid-fusiform, 1-3-septate, (8-)15-29 × (1.5)2.0-2.5(-3.0) $\mu \mathrm{m}$

C. guyanensis

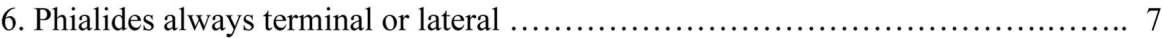

7. Conidia acicular, pale brown, 3-6-septate, $(18-30 \times 1.0-1.2 \mu \mathrm{m})$, phialides short, collarettes hyaline, indistinct, hardly divergent ......................... C. suttonii

7. Conidia of a different shape ................................................. 8

8. Conidia up to $20 \mu \mathrm{m}$ long, subhyaline to pale brown, oblong to cylindrical with rounded ends, $(7-18 \times 1.7-2.3 \mu \mathrm{m}) \ldots \ldots \ldots \ldots \ldots \ldots \ldots \ldots \ldots \ldots \ldots \ldots \ldots \ldots \ldots$. pluriseptata

8. Conidia longer than $20 \mu \mathrm{m}$ long, hyaline .....................................

9. Conidia up to $2 \mu \mathrm{m}$ wide $(30-55 \times 1.2-1.5 \mu \mathrm{m})$, vermiform, $4-8$-septate $\ldots$ C. vermispora

9. Conidia wider than $2 \mu \mathrm{m}$ wide $(25-40 \times 3.5-5.5 \mu \mathrm{m})$, fusiform .................... indica

10. Phialides nearly exclusively intercalary, budding cells present ................ 11

10. Phialides prominent, terminal or lateral, budding cells absent .................... 12

11. Collarettes narrowly funnel-shaped to almost cylindrical, $1.5-2.5 \mu \mathrm{m}$ deep ......C. reptans

11. Collarettes triangular to funnel-shaped, flaring at the opening, $1.5 \mu \mathrm{m}$ deep ......C. sessilis

12. Phialides flask-shaped to elongate, sometimes narrowed toward the tip; collarettes

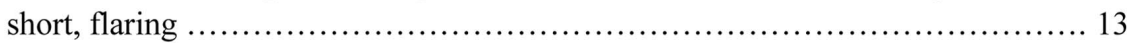

12. Phialides cylindrical, elongate; collarettes slightly diverging, 1.0-2.0 $\mu \mathrm{m}$ deep; conidia clavate to fusiform

13. Conidia subspherical to ellipsoidal $(1.0-2.5 \times 1.0-1.5 \mu \mathrm{m})$.................... europaea

13. Conidia obovoidal, bases broadly truncate $(1.0-2.0 \times 0.8-1.0 \mu \mathrm{m}) \ldots \ldots \ldots \ldots$. . . . ambigua

14. Phialides brown; collarettes cylindrical, hardly diverging; conidia longer than $5 \mu \mathrm{m}$ $(5.0-7.5 \times 1.0-1.7 \mu \mathrm{m})$ C. oxyspora

14. Phialides slightly pigmented; collarettes narrowly funnel-shaped, flaring at the opening; conidia usually not exceeding $5 \mu \mathrm{m}$ in length $(3.0-5.5 \times 1.3-2.2 \mu \mathrm{m})$

C. olivacea

Figure 11. Key to species accepted in Cyphellophora. doi:10.1371/journal.pone.0063547.g011

Comments. Although not closely related, Aphanophora eugeniae and Camptophora hylomeconis group within the Chaetothyriaceae with Exophiala eucalyptorum (Fig. 14) and Vonarxia vagans (Fig. 15) in all three phylogenies (Figs. 1-3). Based on molecular data and their distinctive morphologies, these taxa are excluded from Cyphellophora and introduced as the type species of separate genera.

Aphanophora, Camptophora and E. eucalyptorum grouped constantly together in the ITS, ITS- $\beta$-tubulin and four-gene phylogenies and form predominantly intercalary phialides with minute or inconspicuous hyaline collarettes. Their conidia undergo frequent microcyclic conidiation (Figs. 12I; 13C, D) in axenic culture (and as budding and germinating cells in E. eucalyptorum, Fig. 14E, G). The main differences between Aphanophora and Camptophora lie in the morphology of phialidic loci and presence or absence of conidial segments. In Aphanophora one to three phialidic openings are formed in each intercalary phialide; the collarettes are inconspicuous, and the multiple conidiogenous loci become swollen and may appear subdenticulate [17]. The conidia, which are composed of several constricted segments with a median secondary septum, closely resemble those of $V$. vagans [63] and 


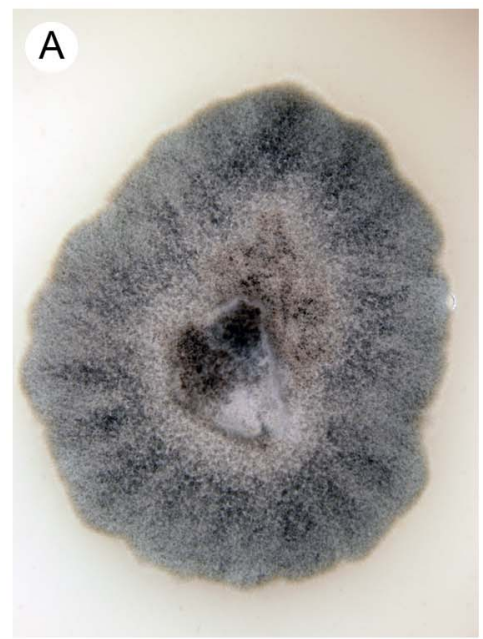

B
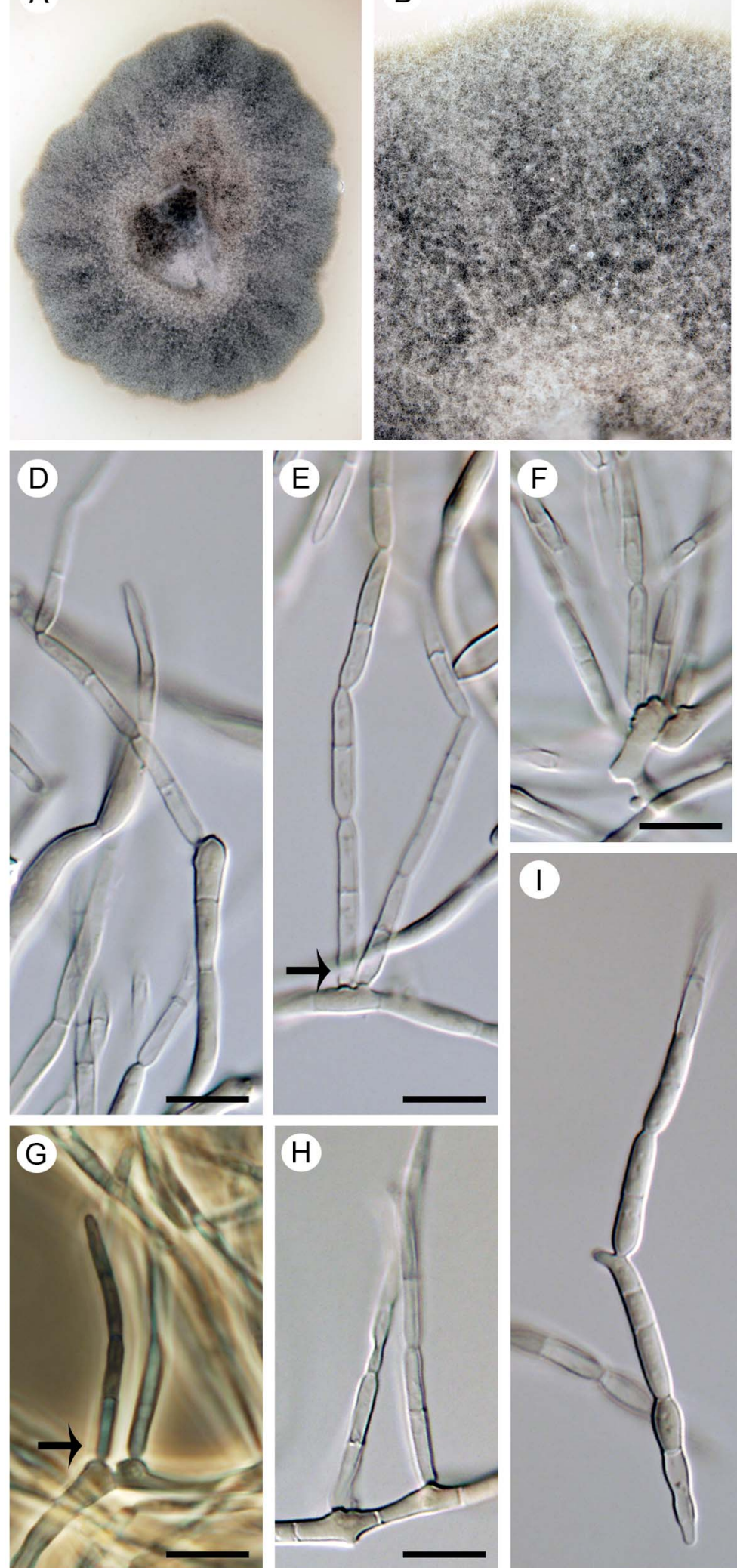
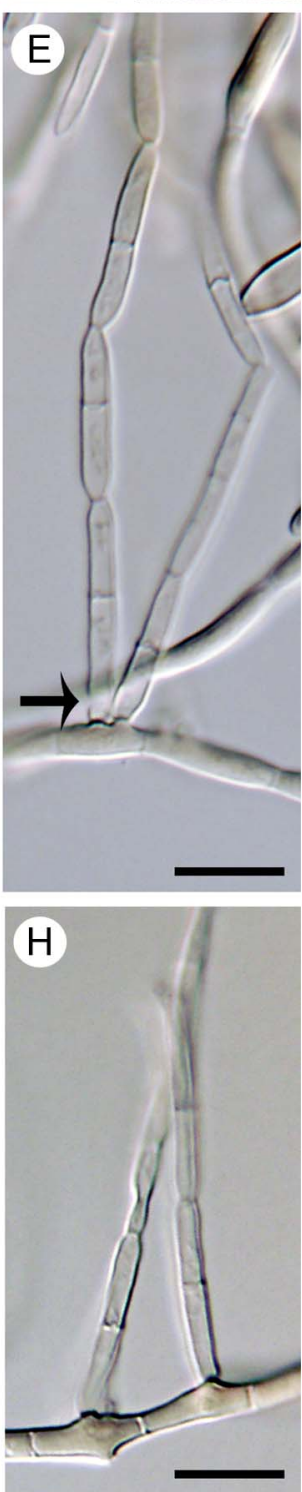

C
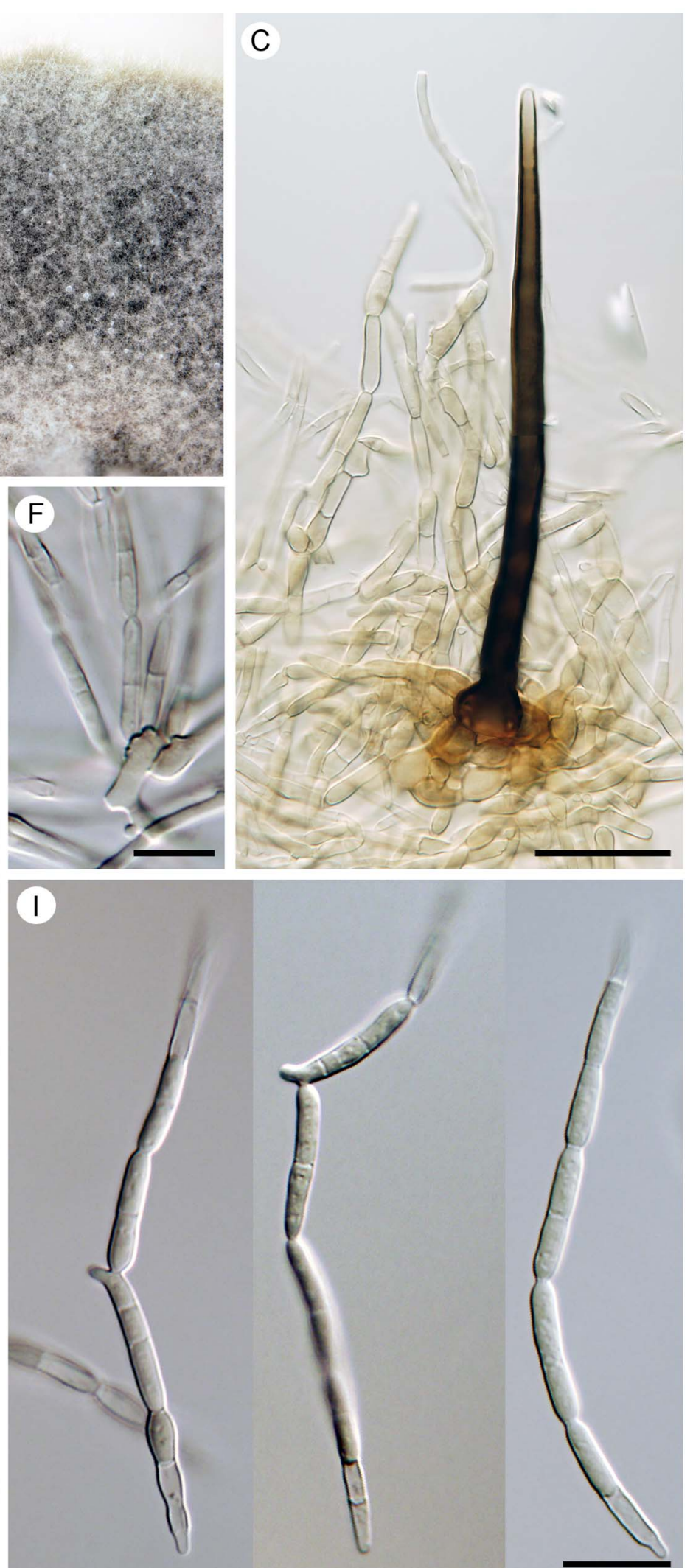

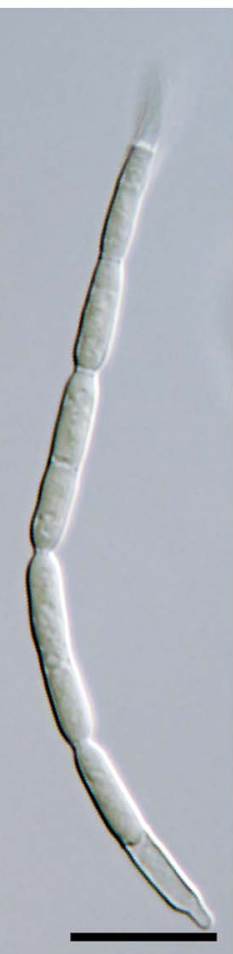

Figure 12. Aphanophora eugeniae, a foliicolous species with conidia forming long one-septate segments. Living culture with a detail of aerial mycelium $(A, B)$, seta that occurs rarely in culture $(C)$, phialides with multiple loci within inconspicuous collarettes (arrow indicates the collarette, D-H), and conidia undergoing microcyclic conidiation (I). DIC (C-F, H, I), PC (G), bar = $20 \mu \mathrm{m}(C), 10 \mu \mathrm{m}(\mathrm{A}, \mathrm{B}, \mathrm{D}-\mathrm{I})$. CBS 124105 (ex-type). doi:10.1371/journal.pone.0063547.g012 


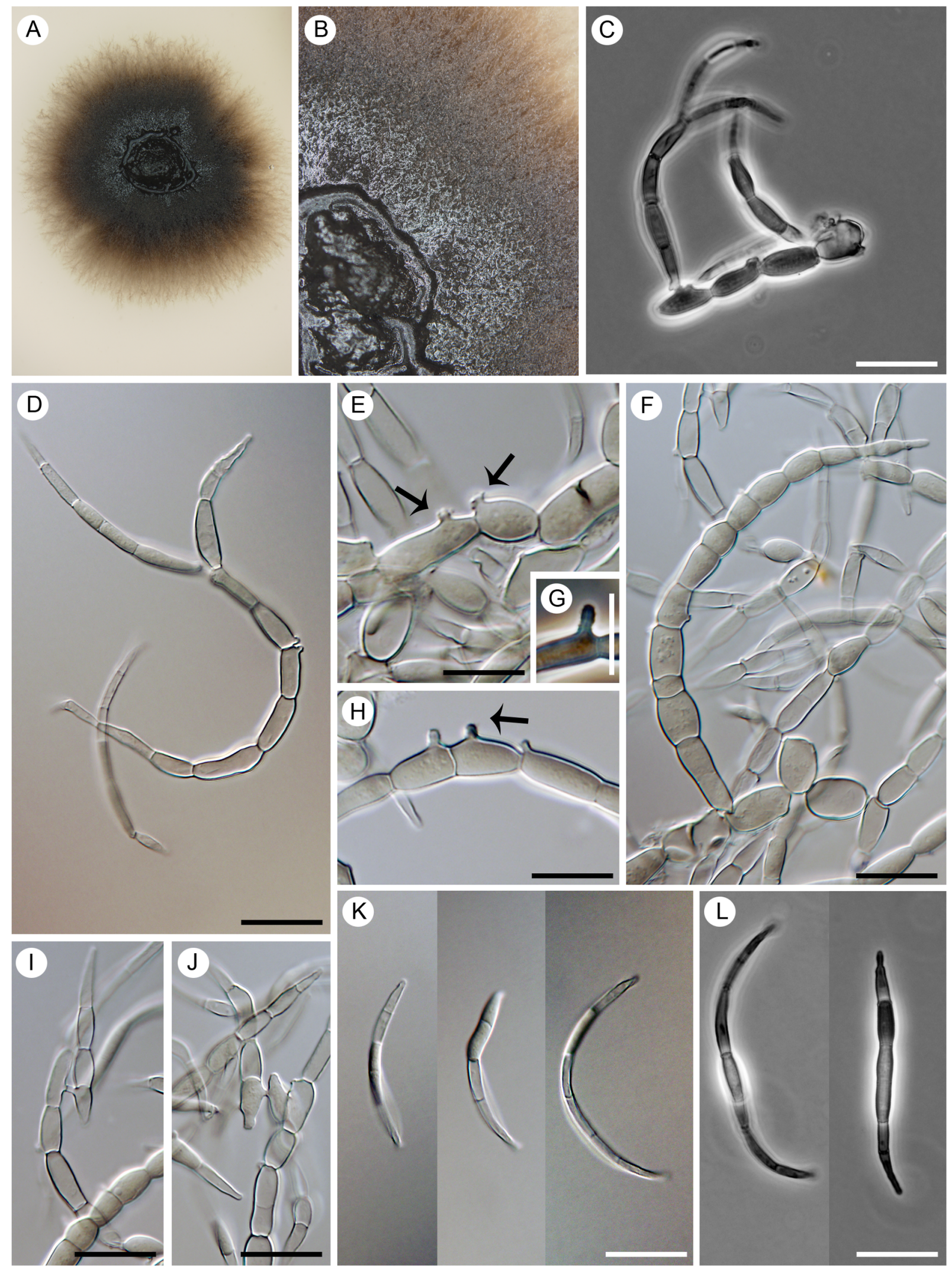

Figure 13. Camptophora hylomeconis, a foliicolous species with sickle-shaped septate conidia. Living culture with a detail of the center of the colony having a yeast-like appearance $(A, B)$, intercalary phialides with conidia $(C, D)$, a detail of conspicuous phialidic loci that can proliferate percurrently above the collarette (arrows indicate the proliferation, E-F), anastomosing conidia (I, J), and sickle-shaped or sigmoid septate conidia (K, L). DIC (D-F, H-K), PC (C, G, L), bar = $10 \mu \mathrm{m}$. CBS 113311 (ex-type).

doi:10.1371/journal.pone.0063547.g013 
A
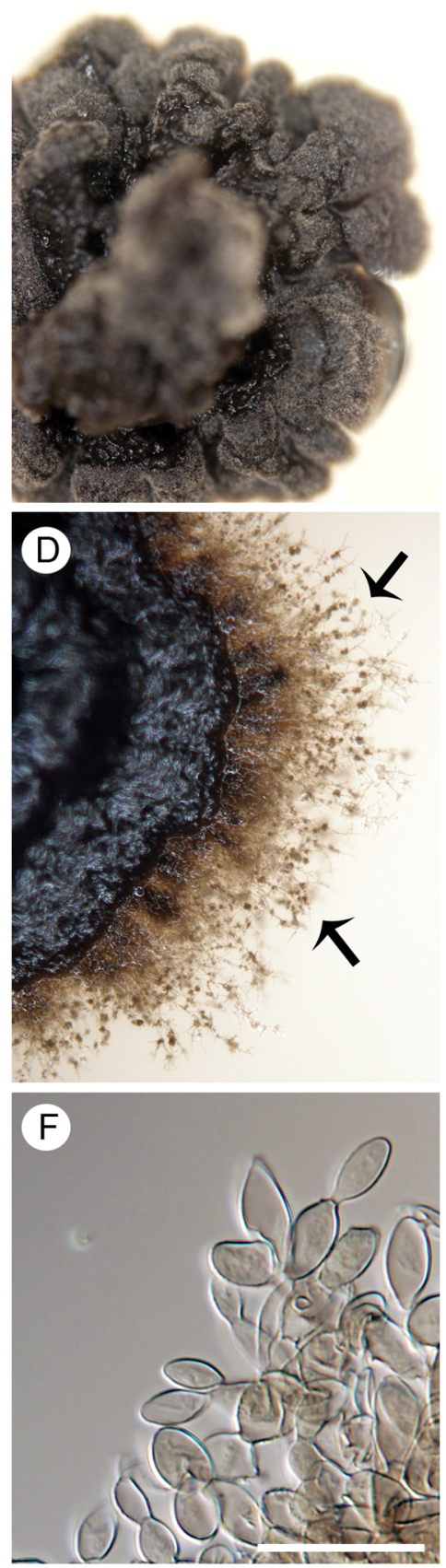

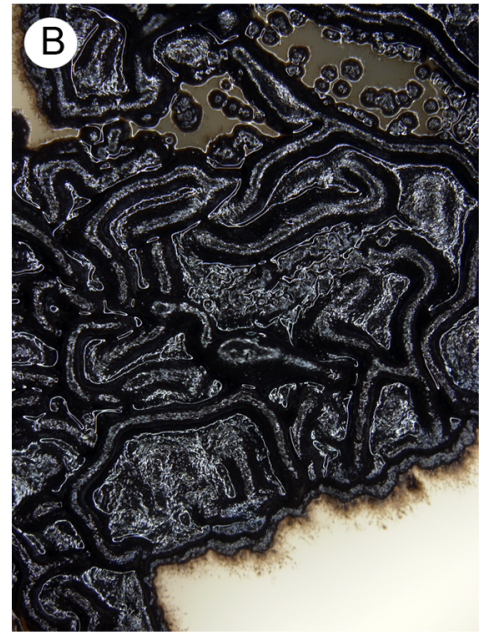

E
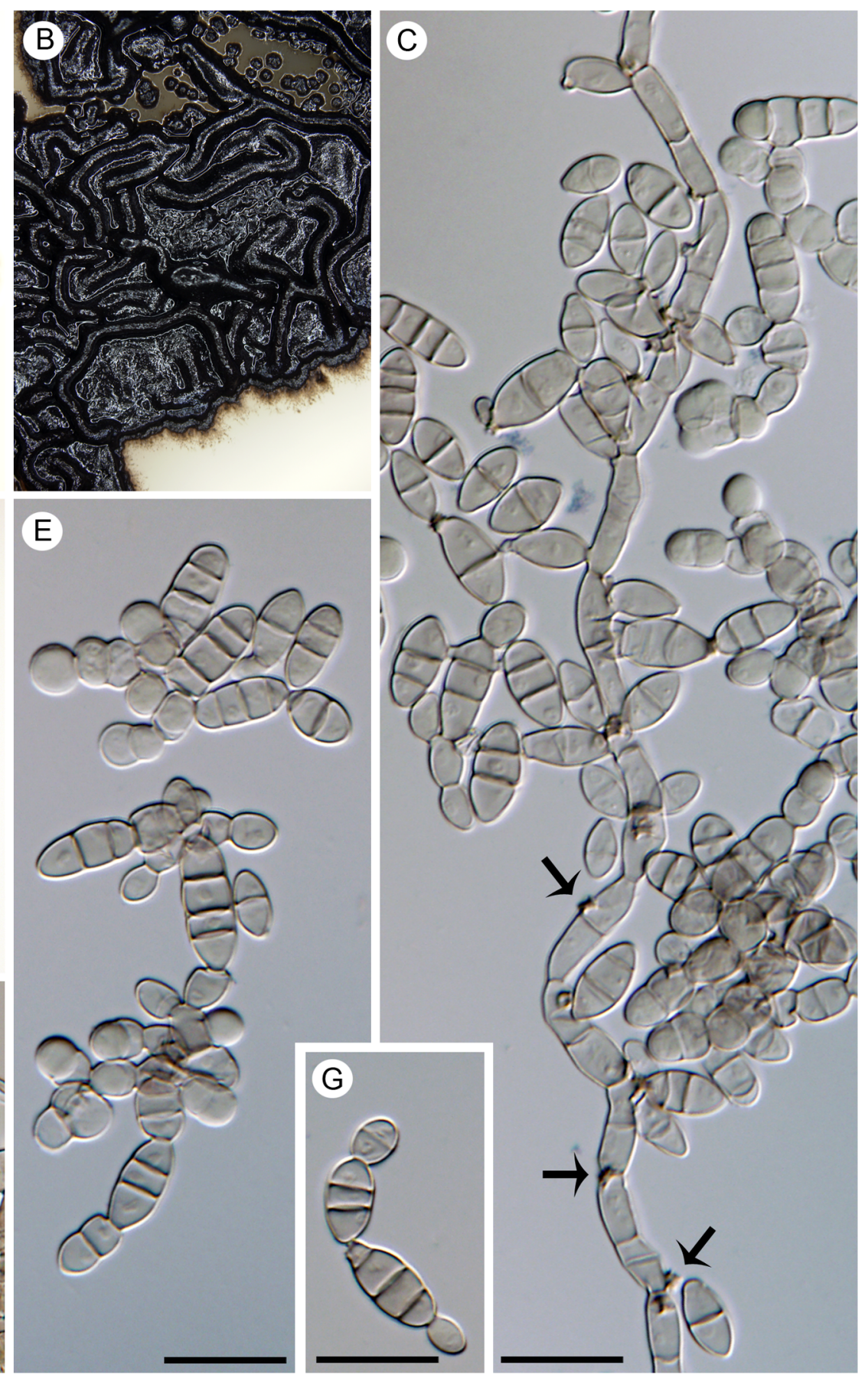

Figure 14. Exophiala eucalyptorum, a dematiaceous hyphomycete with rare hyphal growth in vitro. Living culture with a detail of the colony with sparse aerial hyphae (A) and moist, cerebriform surface (B), hyphae from the margin of the colony with a phialidic opening in every cell $(C, D)$, nonseptate conidia (F; 21 days), and germinating several-septate conidia (arrows indicate proliferating phialidic openings, E, G; 3 months). DIC, bar $=10 \mu \mathrm{m}$. CBS 121638 (ex-type), WUC 637 (only B, D).

doi:10.1371/journal.pone.0063547.g014

anamorphs of Ceramothyrium [64,65]. In contrast, C. hylomeconis produces conspicuous phialidic openings with single conidiogenous loci (rarely two loci) within the collarette that proliferate percurrently. The conidia are (1-)3-5-septate, fusiform to sickleshaped with constricted cell walls, but the conidial segments observed in Aphanophora are not formed.

Knufia peltigerae. (Fuckel) Réblová \& Unter., comb. nov. [urn:lsid:indexfungorum.org:names: 803681]
Basionym. Trichosphaeria peltigerae Fuckel, Jb. nassau. Ver. Naturk. 27-28: 25. 1874 (1873-74).

三 Capronia peltigerae (Fuckel) D. Hawksw., Syst. Ascom. 6: 120. 1987.

Habitat. The ascomata of $K$. peltigerae occur on thalli of species of Peltigera $[66,67,68]$.

Comments. In the ITS and four-gene phylogeny, Capronia peltigerae grouped among species of the genus Knufia. Our results 

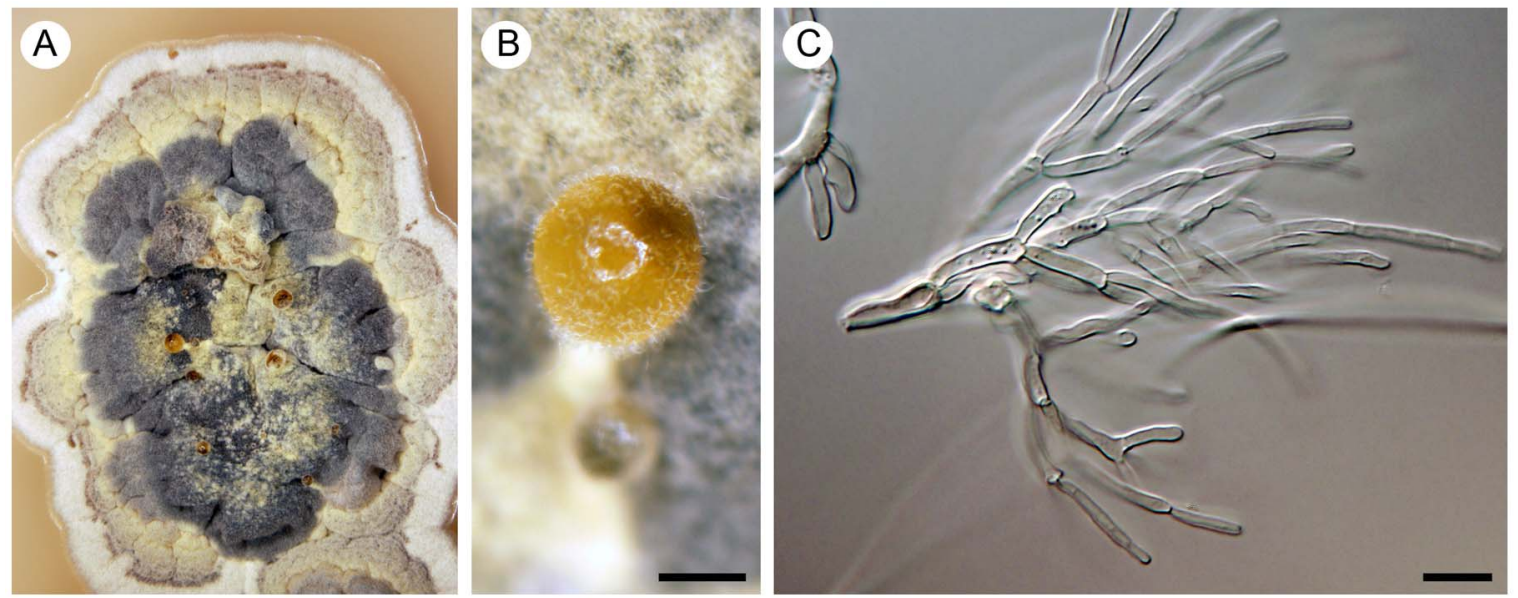

Figure 15. Vonarxia vagans, a foliicolous hyphomycete with blastic conidiogenesis. Living culture with several conidiomata visible in the center of the colony $(A)$ and a detail of the conidioma with conidia extending from the surface of the yellow gelatinous matrix (B), branched mycelium forming conidia at the tips (C). DIC, bar $=1 \mathrm{~mm}$ (B), $10 \mu \mathrm{m}$ (C). CBS 123533 (ex-type).

doi:10.1371/journal.pone.0063547.g015

are in agreement with Untereiner et al. [68], who positioned this species outside the Herpotrichiellaceae among rock-inhabiting strains and other species currently referred to Knufia [37]. In axenic culture, $K$. peltigerae exhibits only filamentous growth and remains sterile. No conidial anamorph has been observed around the ascomata of this species on natural substrates $[67,68]$. Knufia peltigerae represents the only member of the genus with a known teleomorph. In the morphology of its dark, setose ascomata that contain saccate bitunicate asci with hyaline, septate ascospores, it is similar to the known epiphyllous teleomorphs of the Chaetothyriaceae [38,64] and Trichomeriaceae [39].

Phaeococcomyces catenatus. (de Hoog \& Herm.-Nijh.) de Hoog, Taxon 28: 348. 1979.

$\equiv$ Phaeococcus catenatus de Hoog \& Herm.-Nijh., Stud. Mycol. 15: 126. 1977.

= Exophiala placitae Crous \& Summerell, Fungal Planet 17: 1. 2007.

Habitat. The ex-type strain of $P$. catenatus was isolated from the air [58] and the ex-type strain of $E$. placitae formed pycnothyria on leaves of Eucalyptus placita [69].

Comments. In the ITS and four-gene phylogenies (Figs. 1, 3) the ex-type strain of P. catenatus (CBS 650.76) grouped consistently in a strongly supported, monophyletic clade with the ex-type strain of E. placitae (CPC 13707). Based on the morphological similarities of these species in vitro, $E$. placitae is reduced to synonymy of $P$. catenatus. Both fungi produce abundant globose, budding cells in axenic culture that are pale brown, 0 -2-septate, and give rise to new identical cells from 1-6 loci. The cells adhere in torulose, branched or unbranched chains. Hyphae are absent although some short, torulose fragments may recall hyphal growth. Phaecoccomyces catenatus was previously known only in vitro. The strain CPC 13707 (herbarium sample CBS H-19922) represents a first documented case of occurrence of this fungus on natural substratum [69]. In vivo this species forms dark, flat, centrally ostiolate pycnothyria. Conidia are pale brown, globose, 1-celled, and forming up to six torulose arms composed of 5-8 cells, with each cell forming short lateral branches composed of several cells.

\section{Discussion}

\section{Chaetothyriales}

The Chaetothyriales encompass fungi with small, typically setose ascomata, fissitunicate, thick-walled, sessile asci, and hyphomycetous anamorphs and synanamorphs. Ecologically, the order comprises taxa occurring on a variety of substrates, and it includes both saprobic species and opportunistic pathogens of humans and animals. Phylogenetic analyses of five genes supported the recognition of the Chaetothyriales together with the Pyrenulales and Verrucariales as members of the Chaetothyriomycetidae, one of the three subclasses of the Eurotiomycetes $[16,70]$.

Only two of the eight families distinguished in the order by Barr [71,72], the Chaetothyriaceae and Herpotrichiellaceae, have been shown to be closely related to the Eurotiales [73]. The third family Coccodiniaceae, although sometimes still accommodated in the order [74], is more closely allied to the Dothideomycetes [73]. The monotypic Trichomeriaceae, accepted as a part of the Chaetothyriaceae in our study, were introduced recently [39]. Based on molecular and DNA structural data, we introduce the new family Cyphellophoraceae.

\section{Cyphellophoraceae}

The Cyphellophoraceae is a phylogenetically distinct lineage that contains Cyphellophora laciniata, the type species of the genus, six other Cyphellophora, and six species treated previously in Phialophora. Our conclusions are in agreement with those of de Hoog et al. [5], Untereiner et al. [18], and Feng et al. [11] who recognized a strongly supported clade separate from the Herpotrichiellaceae containing numerous strains of C. europaea, C. reptans and C. sessilis.

The core of the family contains four species $(C$. laciniata, $C$. fusarioides, C. suttonii, C. vermispora) that are consistently resolved as sister to the morphologically similar taxon, C. pauciseptata in the ITS and ITS- $\beta$-tubulin phylogenies (Figs. 1, 2). Cyphellophora pauciseptata and C. fusarioides are the only species isolated from clinical sources and they are resolved as the members of the same clade in the four-gene analysis (Fig. 3).

Species of Cyphellophora are distinguished from Phialophora verrucosa (Herpotrichiellaceae) by their cylindrical or flask-shaped elongate or intercalary phialides with hyaline or slightly pigmented collarettes that are usually narrowly funnel-shaped with flaring 

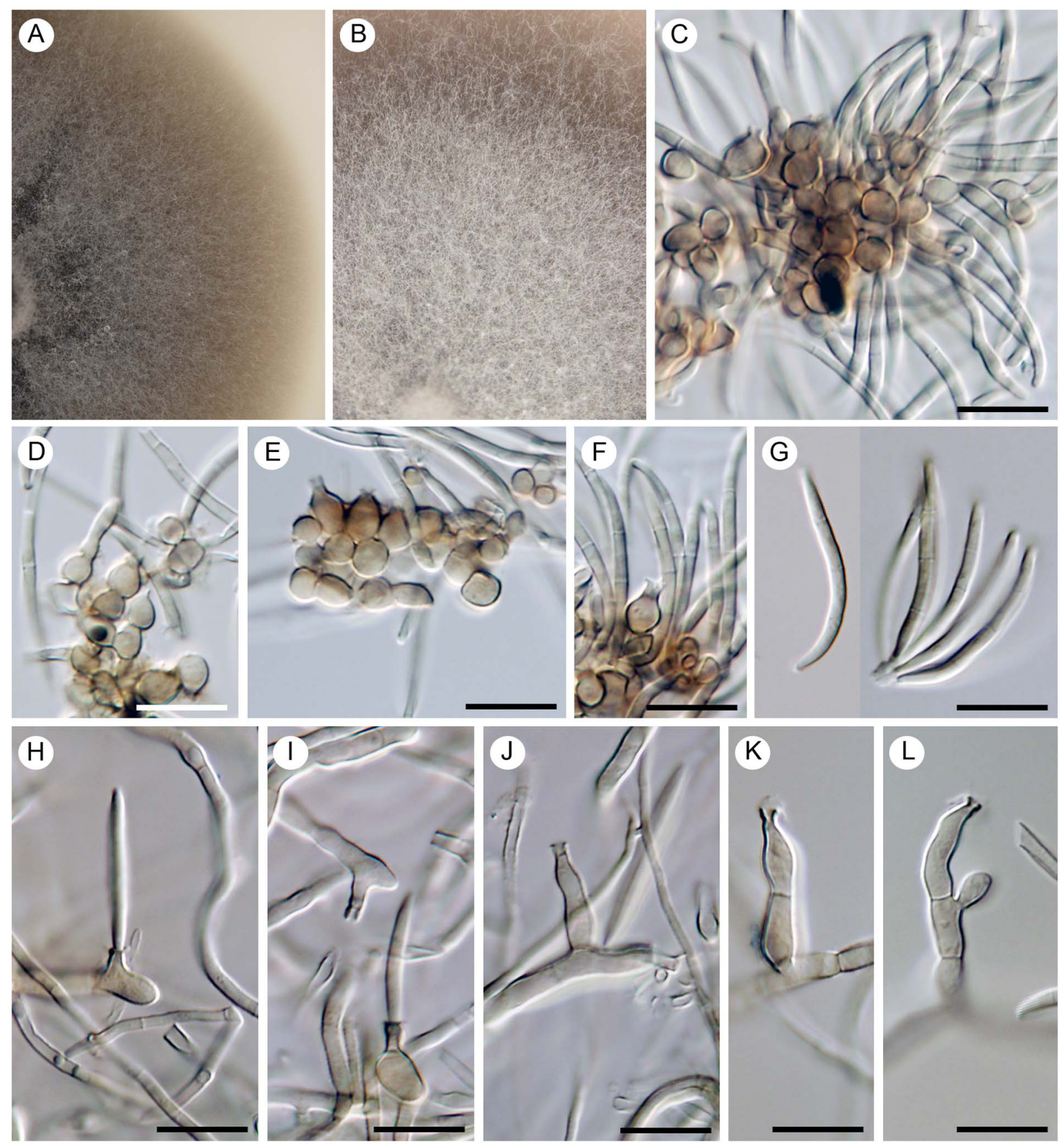

L
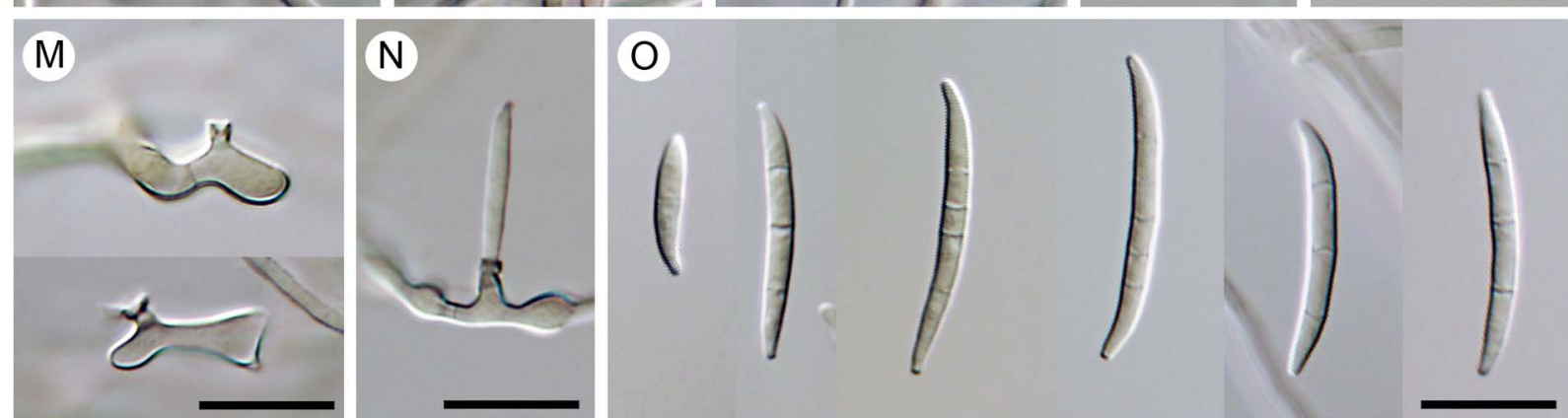

Figure 16. Morphological variability of Cyphellophora guyanensis. A, B, H-O) the ex-type strain of Cyphellophora eucalypti: living culture with a detail of aerial mycelium $(A, B)$, intercalary phialides $(H, I, M, N)$, lateral and terminal cylindrical phialides $(J-L)$, and septate, falcate conidia (O). DIC, bar $=10 \mu \mathrm{m}$. CBS 124764 (ex-type). C-G) the ex-type strain of Cyphellophora guyanensis: lateral flask-shaped phialides aggregated in groups on undifferentiated hyphae (C-F) and conidia (G). DIC, bar = $10 \mu \mathrm{m}$. MUCL 43737 (ex-type).

doi:10.1371/journal.pone.0063547.g016 
opening or almost cylindrical and hardly divergent. In contrast, $P$. verrucosa possesses flask-shaped phialides with broadly funnelshaped or vase-shaped collarettes with flaring openings that are always deeply pigmented and conspicuously darker than the lower part of the phialide. Ten species of Cyphellophora were isolated from skin, nail or other clinical specimens from humans and animals. However, they are clinically distinct from opportunistic pathogens of humans and warm- or cold-blooded animals [4,5,14,24] centered on $P$. verrucosa and other Herpotrichiellaceae that cause chromatoblastomycoses and phaeohyphomycoses. Members of the Herpotrichiellaceae are also known as saprobes on decaying plant material $[75,76]$; a number of these fungi have been isolated from nutrient-poor substrates in extreme environments and are known as rock-inhabiting fungi [77].

Teleomorphs are unknown in the Cyphellophoraceae. The Herpotrichiellaceae accommodates the teleomorph genus Capronia Sacc., which is linked with Phialophora and a number of hyphomycetous anamorphs and synanamorphs that exhibit yeast-like growth in axenic culture. The majority of these anamorphic fungi are species of Cladophialophora Borelli, Exophiala J.W. Carmich., Fonsecaea Negroni, and Rhinocladiella Nannf. $[33,78,79,80,81,82,83]$.

\section{Chaetothyriaceae}

The Chaetothyriaceae were resolved as a robust heterogeneous clade consisting of two subclades in the ITS (99/1.0) and four-gene phylogenies (90/1.0) (Figs. 1, 3). This family, which is also supported by the presence of evolutionarily conserved motifs (M2 and M3) in the ITS2, encompasses saprobic, rock-inhabiting, lichenicolous, epiphytic or biotrophic epiphyllous fungi $[37,38,39,64,65,68,84,85,86]$, that are rarely isolated from clinical samples [87].

The monotypic family Trichomeriaceae [39] is treated here as a part of the Chaetothyriaceae. This lineage is positioned within a large subclade (100/1.0 in ITS and four-gene analyses) that also includes species of Knufia, Brycekendrickomyces acaciae, Cladophialophora proteae, and Phaeococcomyces catenatus. The similarities of the dark, nonstromatic ascomata, sessile, saccate, fissitunicate asci and hyaline, septate ascospores of Knufia peltigerae and species of Trichomerium [39] are remarkable. Species of Knufia, except $K$. peltigerae, have not been linked to teleomorphs. The genus comprises dematiaceous fungi forming sporodochia on the host that contain conidia and multicelullar bodies. In axenic culture, these species produce thallic and blastic conidia from undifferentiated hyphae as well as from phialides; they rarely form endoconidia [37,88]. According to Tsuneda et al. [37] and Gueidan et al. [77] the nuc28S sequence and culture (CBS 268.34) of Glyphium elatum, which grouped in Knufia, are incorrectly determined and we follow their preliminary placement and identification of this strain as Coniosporium sp.

Phaeococcomyces catenatus produces exophiala-like colonies in axenic culture, while cheirospores are formed in pycnothyria on the host (CPG 13707 as Exophiala placitae) [69]. Metulocladosporiella [36] is characterized by its dematiaceous, macronematous conidiophores that form terminal, branched, polyblastic, sympodial conidiogenous cells and conidia that adhere in chains. The monotypic genus Brycekendrickomyces Crous \& M.J. Wingf. grouped on a basal branch in this robust subclade. It was isolated from living leaves of Acacia auriculiformis and produces macronematous, dematiaceous conidiophores with one to several polyblastic conidiogenous cells formed at the tips and hyaline, ellipsoid conidia aggregated in slimy heads [17].

The second subclade included in the Chaetothyriaceae is wellsupported in ITS, ITS- $\beta$-tubulin and four-gene analyses (Figs. 1-3) and comprises three species of Ceramothyrium, the Vonarxia-group (including Aphanophora, Camptophora, and Exophiala eucalyptorum) and Chaetothyrium brischoficola. Members of this clade are filamentous dematiaceous fungi, although the filamentous nature of $E$. eucalyptorum is inconspicuous. In axenic culture, this species forms immersed, septate hyphae at the margins of the colony (Fig. 14D) with intercalary phialides arising from nearly each cell (Fig. 14C). Teleomorphs of Ceramothyrium, Chaetothyrium and Phaeosaccardinula differ from Knufia and Trichomerium in possessing a thin pellicle that covers the ascomata and merges with subiculum on the surface of the host.

With the exception of Phaeosaccardinula fici [38], the members of the Vonarxia-group (Figs. 1-3) are anamorphic fungi. These species are isolated exclusively from living plants or plant litter and are not involved in human or animal infections. Morphologically, this clade is highly variable and includes species producing blastic conidia as well as conidia from phialidic openings. Aphanophora, Camptophora and E. eucalyptorum produce intercalary phialides. Inconspicuous phialidic openings can also be formed on mature conidia, which anastomose and undergo frequent microcyclic conidiation. The phialidic loci can proliferate percurrently above the collarette in the latter two taxa (Figs. 13E, G, H; 14C). Conidiogenesis in $V$. vagans is blastic (Fig. 15C); hyaline conidia consisting of three upper arms on a short main axis are formed sympodially from one to three different loci at the top of a doliform conidiogenous cell $[17,63]$. In fact, each arm is formed by several 1-septate segments marked by constrictions in the wall at the septa. A secondary septum is positioned in the middle of each segment. Anamorphs experimentally linked with Ceramothyrium were originally described in Stanhughesia Constant. [65] and resemble $V$. vagans in conidiogenesis and conidium morphology. The hyaline blastic conidia of Ceramothyrium are L-shaped, consisting of a main septate or nonseptate axis with one or two laterally oriented septate arms composed of nonseptate or one-septate segments. Cylindrical-elongate conidia divided into septate segments also occur in Aphanophora.

Two taxa originally classified in the Chaetothyriaceae, Ceramothyrium thailandicum and Phaeosaccardinula fici [38] were placed in different clades in the four-gene and ITS analyses. In the multilocus analysis, Ceramothyrium thailandicum grouped with two other Ceramothyrium in a weakly supported clade (Fig. 3), but in the preliminary ITS analysis it was positioned outside the Chaetothyriaceae (not shown). The main differences between the ITS2 of $C$. thailandicum (strain MFLUCG 10-0079) and C. carniolicum (strain CBS 175.95), is that the sequence of the former species includes a $\mathrm{H} 3 \mathrm{~A}$ helix formed from eleven bp and five other nucleotides in the end loop. This type of helix was observed only in the Cyphellophoraceae, Herpotrichiellaceae, and selected Chaetothyriaceae (i.e., V. vagans, Metulocladosporiella and Phaeococcomyces). Because the placement of $C$. thailandicum in our preliminary ITS analysis does not agree with the study of Chomnunti et al. [38], this sequence was not included in the final ITS analysis.

Available sequences of Phaeosaccardinula fici (Chaetothyriaceae) (ITS HQ895840; nuc28S HQ895837) [38] derived from the same strain MFLUCG 10-0080 were positioned in different clades. In the preliminary ITS analysis (not shown) this species was positioned as sister to the Chaetothyriaceae. In the four-gene phylogeny (Fig. 3), it was resolved as sister to Vonarxia vagans. Nag Raj [63] observed several immature ascomata on a stroma of $V$. vagans on the host in the type material, LPS 12280 as Kazualia vagans (Speg.) Nag Raj, quoting Spegazzini's handwriting on the label. The suggested link between the two morphs still needs to be confirmed experimentally. However, the grouping of $P$. fici with anamorphic $V$. vagans in one clade in the four-gene phylogeny 
suggests such connection and the existence of a teleomorph for the later taxon. Considering the contradictory phylogenetic relationships and the probable anamorph-teleomorph link between Vonarxia and Phaeosaccardinula, only the nuc28S sequence was included in our study.

\section{Secondary structure of ITS}

Comparison of 2D structures of ITS1 and ITS2 of members of the Chaetothyriales confirmed three major phylogenetic groups at the family level. Within this order we recorded 45 and 64 conserved nucleotides in the three helices of the ITS1 (Fig. 5) and four helices of ITS2 (Figs. 6, 7), respectively. These homologous characters are unevenly distributed in hairpin loops and served as a backbone of the multiple sequence alignment.

The three evolutionary motifs (Table 1) observed in the primary ITS2 sequence were mapped on the ITS phylogram (Fig. 1) and 2D structures of ITS2 (Figs. 6, 7). The M1 motif with three recorded deviations is repeated in all members of the Chaetothyriales. The other two motifs identified in the H3 helix of ITS2 characterize certain clades. The M2 motif is unique for the Cyphellophoraceae (UCUG) and the Herpotrichiellaceae (CGUG), but varies slightly within the Chaetothyriaceae (UCGG), i.e., CGUA in Ceramothyrium carniolicum, UCAG in Cladophialophora proteae and $\mathrm{UCCC}(\mathbf{U})$ among species of Trichomerium. The M3 motif is considered one of the hallmarks of the ITS2 secondary structure [29] and it may indicate family or even higher taxonomic relationships. It defines the Cyphellophoraceae (UGUA), Herpotrichiellaceae (GGUA) and some Chaetothyriaceae (GGUG). The subclade containing the Vonarxia-group, Ceramothyrium and Chaetothyrium is delimited by the GGUG motif and is accepted as the Chaetothyriaceae s. str. It is sister to a robust subclade within the Chaetothyriaceae that includes three different M3 motifs (Fig. 1). The most common motif, GGUG, occurs in the majority of taxa and two unique motifs, UUCG and GAUA, are found in Trichomerium and Phaeococcomyces, respectively. This second subclade may represent several families but we think it is more likely that the M3 motif will be shown to deviate among genera within a single family.

ITS1. - An experimental 3D structure of ITS1 does not exist but study of the 2D structure of the ITSl in eukaryotes using comparative methods reveals an open loop with multiple helices $[89,90,91,92]$. One of these helices (H2, Fig. 5) used to define species complex groups $[93,94]$ was detected in fungi by Réblová and Winka [95] who then employed the 2D structure of $\mathrm{H} 2$ to delimit groups of anamorphs linked with Chaetosphaeria of the Chaetosphaeriaceae.

The consensus 2D structure of ITS1 of members of the Chaetothyriales determined in this study comprises three helices $(\mathrm{H} 1-\mathrm{H} 3)$ and a highly variable region at $3^{\prime}$-end ( $c a$ 60-70 nt) containing many insertions and deletions. We recorded significant variation among Cyphellophora in one of the two CBCs in H1 (Fig. 5) (i.e., $\mathrm{C}=\mathrm{G}$ in the Herpotrichiellaceae, $\mathrm{A}-\mathrm{U}$ in the C. laciniata clade, $\mathrm{G}=\mathrm{C}$ in other Cyphellophoraceae). In five species with $\mathrm{G}=\mathrm{C}$ substitution we observed another change in the primary sequence that resulted in a minor change in the stem structure. Specifically, C. ambigua, C. guyanensis, C. olivacea, C. pluriseptata and C. reptans possess a ' $\mathrm{C}$ ' insertion on the 5 ' side of the helix $\mathrm{H} 1$ that causes a rise of the ' $\mathrm{U}$ ' bulge (Fig. $5 \mathrm{~B}$ ) such that the ' $\mathrm{C}$ ' insertion after $\mathrm{U}$ leads to a preferred formation of a canonical $\mathrm{C}=\mathrm{G}$ pair (followed by the $\mathrm{CBC} A-\mathrm{U} \rightarrow \mathrm{G}=\mathrm{C}$ ) instead of forming a wobble pair $\mathrm{U} / \mathrm{G}$ characteristic of almost all members of the Chaetothyriales.

We observed a G/G non-canonical pair in the right arm of helix $\mathrm{H} 3$ in all members of the Cyphellophoraceae (Fig. 5B), it always occurs after the third base pair. This mismatch does not occur in the Herpotrichiellaceae (where $2 \times 2$ symmetrical loop exists) and other members of the Chaetothyriales. In two species formerly included in Cyphellophora (Aphanophora eugeniae and Camptophora hylomeconis) it changes to a wobble pair (G/U).

Considering the number of $\mathrm{CBC}$ s and hCBCs in the three helices of ITS1 and comparing them to the four helices of ITS2, the 2D structure of ITS1 seems more conservative among members of the Herpotrichiellaceae, Cyphellophoraceae and Vonarxia-group.

ITS2. - As with the ITS1, the 3D structure of ITS2 has not been determined. However, the 2D structure of the ITS2 has been investigated [30,91,96,97,98,99]. The number of four helices represents one of the hallmarks and is universal among eukaryotes. Additional helices may be positioned on the ring structure anywhere between helices $1-4$ in different groups of organisms [29]. This feature was observed in the Chaetothyriales. A short helix labeled H3A (Figs. 6, 7) was recognized in the Herpotrichiellaceae, Cyphellophoraceae and three Chaetothyriaceae. The basal part of H3A consists of three bp with co-evolving nucleotides (CBCs), while the upper part is highly variable and includes an internal loop and additional base pairs.

Only the Herpotrichiellaceae possess four basal bp in helix $\mathrm{H} 1$ (Fig. 6). In the Cyphellophoraceae and Vonarxia-group, two nonCBCs changes occur in the second and third bp resulting in the disruption of the duplex (Fig. 7B). The $\mathrm{Hl}$ helix of these taxa is shorter with longer adjacent one-stranded regions. Helices $\mathrm{H} 2$ and H3 of ITS2 are most variable among the Cyphellophoraceae. The pyrimidine-pyrimidine mismatch of $\mathrm{H} 2$ was not present in all species and the predicted models of 2D structure of ITS2 revealed this region only in C. europaea $(\mathrm{U} / \mathrm{U}, \mathrm{U} / \mathrm{C})$, C. oxyspora $(\mathrm{U} / \mathrm{U}, \mathrm{C} / \mathrm{C})$ and $C$. reptans $(\mathrm{C} / \mathrm{C})$. Although helices $\mathrm{H} 1$ and $\mathrm{H} 4$ of the ITS2 were considered the most variable regions at species levels among eukaryotes [29], these regions are more or less conserved within the Chaetothyriales.

\section{Changes in $\mathrm{CBCs}$ and $\mathrm{hCBCs}$ as a tool to improve alignments and to study evolutionary history of ITS1 and ITS2}

Examination of all positions in ITS1 $(\mathrm{H} 1-\mathrm{H} 3)$ and ITS2 (H1$\mathrm{H} 4)$ revealed that some pairs with double-sided $(\mathrm{CBC})$ and onesided ( $\mathrm{hCBC}$ ) substitutions uniquely characterize certain clades and represent non-homoplasious synapomorphies within the Chaetothyriales (Figs. 4, 6, 7). In H1-H3 of ITS1 only five base pairs that always display CBCs and thirteen that contained hCBCs or both CBCs/hCBCs were discerned. Of these, two CBCs and one hCBCs are unique for the Cyphellophoraceae. Ten base pairs in the entire ITS2 molecule displayed both GBCs and hCBCs, while only six pairs displayed CBCs; only eight of the former and three of the latter were recorded in the conserved regions of $\mathrm{H} 2$ and $\mathrm{H} 3$ helices. Of these, only one $\mathrm{CBC}$ and one hCBC are unique to the Cyphellophoraceae and one CBC characterizes the Vonarxia-group in the Chaetothyriaceae s. str. Another base pair of the five in H3, where non-CBCs were observed, is unique to the Vonarxia-group.

We observed that GBC and hCBC substitutions are not equally distributed in ITS of the lineages resolved in the Chaetothyriales. Caisová et al. [97] proposed that $\mathrm{CBC}$ and hCBC substitutions have evolved independently and thus hCBCs do not contribute to the origin of CBCs (i.e., hCBCs are not the intermediate steps between $\mathrm{G}=\mathrm{C} \leftrightarrow \mathrm{G} / \mathrm{U} \leftrightarrow \mathrm{A}-\mathrm{U})$. Since a $\mathrm{G}=\mathrm{C}$ pair with three hydrogen bonds is more stable than $\mathrm{A}-\mathrm{U}$ and $\mathrm{G} / \mathrm{U}$ pairs with just two hydrogen bonds [100], $\mathrm{G}=\mathrm{C}$ contributes more to the folding and stability of RNA transcripts than A-U and wobble G/U pairs, even though an important role in stability of RNA molecules is 
also played by stacking interactions [101]. Caisová et al. [97] suggested that two-sided substitutions leading to the formation of canonical $\mathrm{G}=\mathrm{C}, \mathrm{C}=\mathrm{G}$ pairs, via short-lived non-paired intermediates (i.e., $\mathrm{N}=\mathrm{N} \leftrightarrow \mathrm{N} / \mathrm{N} \leftrightarrow \mathrm{N}-\mathrm{N}$ ), may occur in organisms undergoing specialization to certain habitats (e.g. higher temperatures). The rapidly evolving hCBCs substitutions, which occur more frequently in taxa, may also facilitate faster ecological adaptations of organisms followed by changes in morphology. These adaptations may involve changes of substrate.

This hypothesis is supported in our study among phylogenetically closely related members of the Chaetothyriales. For example, hCBCs and pairs with non-compensating changes in conserved areas of $\mathrm{H} 2$ and $\mathrm{H} 3$ of the ITS2 vary significantly within Cyphellophora. We observed five pairs in the $\mathrm{H} 2$ that show canonical $\mathrm{G}=\mathrm{C}, \mathrm{C}=\mathrm{G}$ pairs in majority Cyphellophora, but several species are characterized by a wobble G/U, U/G pairs. NonCBCs pairs occur in Cyphellophora with lower frequency than the hCBCs; in three Cyphellophora with non-septate conidia we discerned two non-CBCs substitutions (the pyrimidine-pyrimidine mismatch of $\mathrm{H} 2$ ) while other species possess canonical A-U, $\mathrm{G}=\mathrm{C}$ pairs at these positions. In contrast, among members of the Chaetothyriales the H3 of ITS2 contains five base pairs with nonCBCs and three with hCBCs. Two of the former and two of the latter are the sources of the greatest infrageneric variability of Cyphellophora. In addition, we distinguished two pairs exhibiting CBCs, hCBCs and non-CBCs (both in H3) in ITS1 that occur only in the Cyphellophoraceae and characterize individual species. We assume the occurrence of non-CBCs and hCBCs substitutions in Cyphellophora at certain base pairs indicate broad ecological adaptations that are reflected as saprobic (considering a wide range of substrates) or parasitic life-styles of these species.

Two newly segregated monotypic genera in the Vonarxia-group, Aphanophora and Camptophora, differ from Cyphellophora in five (H2) and eight (H3) pairs showing CBCs, hCBCs and non-CBCs substitutions in ITS2, respectively. Six pairs with changes were observed between Cyphellophora and both segregated genera (i.e., two CBCs, two hCBCs and two non-CBCs). Six different substitutions (i.e., two $\mathrm{CBC}$, three hCBCs and one non-CBC) occur only between Cyphellophora and Camptophora and only one base pair with hCBC is unique between Cyphellophora and Aphanophora.

The CBC species concept, which has been used to delimit biological species, is based on co-evolution of nucleotides in the most conserved helices (H2 and H3) of the ITS2 $[30,31,102,103,104]$. Since even a single CBC in H2 and H3 of the ITS2 indicates sexual incompatibility, the original hypothesis defined a CBC clade as including organisms lacking CBCs in these conserved helices that differ from other $\mathrm{CBC}$ clades by as little as a single CBC. In contrast, CBCs in $\mathrm{H} 1$ and $\mathrm{H} 4$ of ITS2, and hCBCs in $\mathrm{H} 2$ and $\mathrm{H} 3$ may still allow mating between two organisms and therefore are less useful in species delimitation [31]. Also, it is important to note that the absence of CBC between two organisms does not indicate that they belong to different species [72]. CBC clades usually fall into one or more $\mathbf{Z}$ clades encompassing groups of organisms producing compatible gametes that can form zygotes [31], but which are separated by various pre- and postzygotic isolation mechanisms. Therefore, the $\mathrm{Z}$ clades rather than the CBC clades contain 'biological species' or represent a diverging population of one to several morphotypes capable of interbreeding.

The CBC hypothesis has been tested in Chlorophyta and representatives of the Fagales $[31,105,106]$ and also by employing up to 100,000 2D models from the ITS2 database $[52,107,108,109]$. In-depth analysis of the ITS2 of members of the Ulvales (Chlorophyta) revealed a number of discrepancies between the proposed CBC concept and the evolution of the ITS2 [97] and it was concluded that careful analysis of ITS2 evolution and phylogeny is required before species are proposed based on the presence or absence of CBCs.

Because our data sets contained a limited number of strains of each species we could not investigate the CBC species concept in each clade at a level that would resolve differences between closely related taxa or indicate cryptic species. However, recording all observed substitutions and mapping them onto the 2D structure of ITS1 and ITS2 allowed us to significantly improve the alignment of ITS sequences of members of the Chaetothyriales.

The existence of two $\mathrm{Z}$ clades within Cyphellophora guyanensis (Fig. 16) is suggested by differences in the 2D structure of the conserved part of ITS2 of the isolates found in this lineage. This clade contained sequences of five strains; four of C. guyanensis, including the ex-type strain (MUCL 43737), and the ex-type strain of C. eucalypti Cheewangkoon \& Crous (CBS 124764). These species are morphologically similar, and $C$. eucalypti has been treated recently as a synonym of $C$. guyanensis based on the comparison of sequence data [11]. However, Feng et al. [11] did not include the ex-type strain of $C$. guyanensis in their analyses. In our ITS and ITS- $\beta$-tubulin phylogenies, all five strains formed a well-supported $\mathrm{CBC}$ clade with two $\mathrm{Z}$ clades, each of which contained an ex-type strain. In the ITS phylogeny (Fig. 1), the extype strain of C.eucalypti (CBS 124764) was positioned as separate from the subclade that included four strains of $C$. guyanensis. In the ITS- $\beta$-tubulin analysis (Fig. 2) the CBC clade was again divided into two subclades; one containing the ex-type strain of $C$. guyanensis (MUCL 43737) and the other comprising the remaining isolates of this species. We observed the following differences in the ITS1 and ITS2 between the ex-type strain of C. guyanensis and the other members of this clade: 1) the last two bp differ in the right arm of helix H3 of ITS1 of MUCL 43737; the last bp is missing due to a non-CBC change $(\mathrm{U}-\mathrm{A} \rightarrow \mathrm{U} / \mathrm{C})$ resulting in a longer loop and the second last bp is changed to a wobble pair $(\mathrm{C}=\mathrm{G} \rightarrow \mathrm{U}$ / $\mathrm{G})$, (2) the tenth bp with canonical $\mathrm{G}=\mathrm{C}$ pair of MUCL 43737 is changed to a wobble pair G/U in helix H2 of ITS2, and 3) MUCL 43737 has the longest hairpin loop (i.e., 5 nt vs. 3 nt) in the H3A helix of ITS2. In contrast, the ITS2 sequence of the ex-type strain of C. eucalypti (CBS 124764) possesses a hairpin loop in H4 that is longer by one nucleotide than that of all other strains in the clade. The grouping of strains in two $\mathrm{Z}$ clades is also supported by morphological differences. The ex-type strain of C. guyanensis [13] lacks intercalary phialides but forms prominent lateral phialides that are often aggregated in large groups (Fig. 16C-F), while $C$. eucalypti [91] is distinguished by the occasional presence of intercalary or short phialides without septa (Fig. 16H, I, M, N).

None of the differences recorded in the 2D structure of ITS1 and in the conserved part of ITS2 warrant recognition of two biological species according to the CBC species concept sensu Coleman [31] and the presence of two hCBCs between the extype strains of this clade indicate that they can theoretically interbreed. Whether these differences correspond with the process of speciation or adaptation to a new niche should be further investigated based on the examination of additional strains exhibiting the morphological characteristics of the ex-type strain of C. eucalypti. The strain MUCL 43737 is distant from other members of this clade in the four-gene ML analysis (Fig. 3) but in the BI analysis the branches of the clade (1.0) collapse. Because the main difference between the two species lies in the occasional occurrence of intercalary phialides in C. eucalypti (the shape and size of phialides and conidia of all isolates in this clade are 
comparable), we accept C. guyanensis as a name for the whole clade. The morphological variability among strains is noted in the key.

\section{Conclusions}

Two novel evolutionary lineages were revealed in the Chaetothyriales based on the analysis of molecular, morphological and ITS structural data. The Cyphellophoraceae, introduced in our study, was consistently resolved as a robust clade based on analyses of ITS, other two ribosomal and three protein-coding gene sequences. The family accommodates dematiaceous microscopic fungi that are known to reproduce asexually only and includes medically important and saprobic fungi occupying a wide range of substrates. We emend Cyphellophora to include species with septate and nonseptate conidia formed on phialidic loci on phialides that can be intercalary, lateral or terminal and propose six new combinations in the genus. A second novel lineage was revealed within the Chaetothyriaceae, and is recognized here as the Vonarxia-group, it includes two Cyphellophora (C. eugeniae and $C$. hylomeconis), Exophiala eucalyptorum and Vonarxia vagans. Based on their distinctive morphologies and molecular data, the former Cyphellophora are introduced as type species of Aphanophora and Camptophora. Capronia peltigerae, known from lichen thalli and recently excluded from the Herpotrichiellaceae, was positioned with rock-inhabiting strains and other species currently referred to Knufia. We propose a new combination for this taxon in Knufia.

Phylogenetic results were supported by analyses of the secondary structure of the ITS1 and ITS2, and the identification of two evolutionary motifs (M2, M3) in the helix H3 of ITS2 molecule that characterize clades recognized as families. We also detected several double-sided (CBCs) and one-sided (hCBCs)

\section{References}

1. Medlar JM (1915) A new fungus, Phialophora verrucosa, pathogenic for man. Mycologia 7: 200-203.

2. Iwatsu T, Miyaji M (1977) Subcutaneous cystic granuloma caused by Phialophora verrucosa. Mycopathologia 64: 165-168.

3. Haase G, Sonntag L, Melzer-Krick B, de Hoog GS (1999) Phylogeny inference by SSU gene analysis of members of the Herpotrichiellaceae with special reference to human pathogenic species. Stud Mycol 43: 80-97.

4. Hoog GS de, Guarro J, Gené J, Figueras MJ (2000) Atlas of clinical fungi 2nd ed. Centraalbureau voor Schimmelcultures, Utrecht and Universitat Rovira I Virgili, Reus. 1124 p.

5. Hoog GS de, Mayser P, Haase G, Horré R, Horrevorts AM (2000) A new species, Phialophora europaea, causing superficial infections in humans. Mycoses 43: 409-416.

6. Vries GA de (1962) Cyphellophora laciniata nov. gen. nov. sp. and Dactylium fusarioides Fragoso et Ciferri. Mycopat Mycol Appl 16: 47-54.

7. Walz A, Hoog GS de (1987) A new species of Cyphellophora. Anton Leeuw Int J G 53: 143-146.

8. Ajello L, Padhye AA, Payne M (1980) Phaeohyphomycosis in a dog caused by Pseudomicrodochium suttonii sp. nov. Mycotaxon 12: 131-136.

9. Vries GA de, Elders M, Luykx MHF (1986) Description of Cyphellophora pluriseptata sp. nov. Anton Leeuw Int J G 52: 141-143.

10. Sutton BC, Campbell CK, Goldschmied-Reouven A (1991) Pseudomicrodochium fusarioides sp. nov. isolated from human bronchial fluid. Mycopathologia 114: $159-161$

11. Feng P, Lu Q, Najafzadeh MJ, Gerrits van den Ende AHG, Sun J, et al. (2012) Cyphellophora and its relatives in Phialophora: biodiversity and possible role in human infection. Fung Diver. Available: DOI 10.1007/s13225-012-0194-5.

12. Nunes AT, Cavalcanti MA, Queiroz Aciole de L (1999) Occurrence of Pseudomicrodochium suttonii in Brazil. Rev Microbiol 30: 52-53.

13. Decock C, Delgado-Rodríguez G, Buchet S, Seng JM (2003) A new species and three new combinations in Cyphellophora, with a note on the taxonomic affinities of the genus, and its relation to Kumbhamaya and Pseudomicrodochium. Anton Leeuw Int J G 84: 209-216.

14. Crous PW, Schubert K, Braun U, Hoog GS de, Hocking AD, et al. (2007) Opportunistic, human-pathogenic species in the Herpotrichiellaceae are phenotypically similar to saprobic or phytopathogenic species in the Venturiaceae. Stud Mycol 58: 185-217.

15. Jacob M, Bhat DJ (2000) Two new endophytic fungi from India. Cryptogamie Mycol 21: 81-88. substitutions in ITS1 and in the conserved regions of helices H2 and H3 of ITS2 that uniquely characterize the Cyphellophoraceae and taxa included in the Vonarxia-group, and which represent nonhomoplasious synapomorphies in the Chaetothyriales.

The molecular and structural RNA data were combined with morphological and cultural characteristics to delimit the novel taxonomic groups. The morphology-based key is provided to facilitate identification of microscopic fungi accepted in the Cyphellophoraceae.

\section{Supporting Information}

Table S1 A list of fungi, isolate information and new sequences determined for this study and those retrieved from GeneBank. (DOG)

\section{Acknowledgments}

We are grateful to Walter Gams for his suggestions regarding the nomenclature of these fungi and other valuable comments. We thank Richard Summerbell for his suggestions and discussion regarding the nondermatophytic filamentous fungi and procedures for their detection. Václav Štépánek and Mesfin Bogale are thanked for generating a number of the sequences used in this study.

\section{Author Contributions}

Conceived and designed the experiments: MR WAU KR. Performed the experiments: MR WAU KR. Analyzed the data: MR WAU KR. Contributed reagents/materials/analysis tools: MR WAU KR. Wrote the paper: MR WAU KR.

16. Geiser DM, Gueidan C, Miadlikowska J, Lutzoni F, Kauff F, et al. (2006) Eurotiomycetes: Eurotiomycetidae and Chaetothyriomycetidae. Mycologia 98: 1053-1064.

17. Crous PW, Braun U, Wingfield MJ, Wood AR, Shin HD, et al. (2009) Phylogeny and taxonomy of obscure genera of microfungi. Persoonia 22: 139 161 .

18. Untereiner WA, Angus A, Réblová M, Orr M-J (2008) The systematics of the Phialophora verrucosa complex: new insights from $\beta$-tubulin, large subunit nuclear rDNA and ITS sequence data. Botany 86: 742-750.

19. Gams W, Holubová-Jechová V (1976) Chloridium and some other dematiaceous hyphomycetes growing on decaying wood. Stud Mycol 13: 1-99.

20. Constantinescu O, Holm K, Holm J (1995) Teleomorph-anamorph connections in Ascomycetes: the anamorphs of three species of Chaetosphaeria. Mycol Res 99: 585-592.

21. Untereiner WA, Naveau FA, Bachewich J, Angus A (2006) Evolutionary relationships of Hyphodiscus hymeniophilus (anamorph Catenulifera rhodogena) inferred from $\beta$-tubulin and nuclear ribosomal DNA sequences. Can J Bot 84: 243-253.

22. Bogale M, Orr M-J, O'Hara MJ, Untereiner WA ( 2010) Systematic of Catenulifera (anamorphic Hyaloscyphaceae) with an assessment of the phylogenetic position of Phialophora hyalina. Fung Biol 114: 396-409.

23. Réblová M, Gams W, Seifert KA (2011) Monilochaetes and allied genera of the Glomerellales, and a reconsideration of families in the Microascales. Stud Mycol 68: $163-191$.

24. Hoog GS de, Weenink O, Gerrits van den Ende AHG (1999) Taxonomy of the Phialophora verrucosa complex with the description of two new species. Stud Mycol 43: 107-122

25. Caretta G, Tosi S, Piontelli E, Hoog de GS (2006) Phialophora sessilis, a lithobiont fungus. Mycotaxon 95: 281-284.

26. Côté CA, Greer CL, Peculis BA (2002) Dynamic conformational model for the role of ITS2 in pre-rRNA processing in yeast. RNA 8: 786-797.

27. Mai JC, Coleman AW (1997) The internal transcribed spacer 2 exhibits a common secondary structure in green algae and flowering plants. J Mol Evol 44: $258-271$.

28. Joseph N, Krauskopf E, Vera MI, Michot B (1999) Ribosomal internal transcribed spacer 2 (ITS2) exhibits a common core of secondary structure in vertebrates and yeast. Nucleic Acids Res 27: 4533-4540.

29. Coleman AW (2007) Pan-eukaryote ITS2 homologies revealed by RNA secondary structure. Nucleic Acids Res 35: 3322-3329. 
30. Coleman AW, Mai JC (1997) Ribosomal DNA ITS-1 and ITS-2 sequence comparisons as a tool for predicting genetic relatedness. J Mol Evol 45: 168177.

31. Coleman AW (2000) The significance of a coincidence between evolutionary landmarks found in mating affinity and a DNA sequence. Protist 151: 1-9.

32. Gams W, Hoekstra ES, Aptroot A (1998) CBS course of mycology, 4th edn. Baarn, The Netherlands: Centraalbureau voor Schimmelcultures. $165 \mathrm{p}$.

33. Untereiner WA, Straus NA, Malloch D (1995) A molecular-morphotaxonomic approach to the systematics of the Herpotrichiellaceae and allied black yeasts. Mycol Res 99: 897-913

34. Lee SB, Taylor JW (1990) Isolation of DNA from fungal mycelium and single spores. In: Innis MA, Gelfand DH, Snisky JJ, White TJ (eds.) PCR protocols: a guide to methods and applications. Academic, San Diego. 282 287.

35. Schmitt I, Crespo A, Divakar PK, Frankhauser JD, Herman-Sackett E, et al. (2009) New primers for promising single-copy genes in fungal phylogenetics and systematics. Persoonia 23: 35-40.

36. Crous PW, Schroers H-J, Groenewald JZ, Braun U, Schubert K (2006) Metulocladosporiella gen. nov. for the causal organism of Cladosporium speckle disease of banana. Mycol Res 110: 264-275.

37. Tsuneda A, Hambleton S, Currah RS (2011) The anamorph genus Knufia and its phylogenetically allied species in Coniosporium, Sarcinomyces, and Phaeococcomyces. Botany 89: 523-536.

38. Chomnunti P, Ko Ko TW, Chukeatirote E, Cai L, Jones EBG, et al. (2012) Phylogeny of Chaetothyriaceae in northern Thailand including three new species. Mycologia 104: 382-395.

39. Chomnunti P, Bhat DJ, Gareth Jones EB, Chukeatirote K, Bahkali AH, et al. (2012) Trichomeriaceae, a new sooty mould family of Chaetothyriales. Fung Diver 56: 63-76.

40. Hall TA (1999) BioEdit 5.0.9: a user-friendly biological sequence alignment editor and analysis program for Windows 95/98/NT. Nuc Acids Symp Ser 41: 95-98.

41. Gutell RR (1993) Collection of small subunit (16 S- and 16 S-like) ribosomal RNA structures. Nucleic Acids Res 21: 3051-3054.

42. Gutell RR, Gray MW, Schnare MN (1993) A compilation of large subunit (23 $\mathrm{S}$ and 23 S-like) ribosomal RNA structures. Nucleic Acids Res 21: 3055-3074.

43. Réblová M, Réblová K (2012) RNA secondary structure, an important bioinformatics tool to enhance multiple sequence alignment: a case study (Sordariomycetes, Fungi). Mycol Prog. Available: DOI 10.1007/s11557-0120836-8.

44. Nylander J (2008) MrModeltest2 v. 2.3. C program for selecting DNA substitution models using PAUP*

45. Stamatakis A, Ludwig T, Meier H (2005) RaxML-III: a fast program for maximum likelihood-based inference of large phylogenetic trees. Bioinformatics 21: 456-463.

46. Stamatakis A (2006) RAxML-VI-HPC: maximum likelihood-based phylogenetic analyses with thousands of taxa and mixed models. Bioinformatics 22: 2688-2690

47. Huelsenbeck JP, Ronquist F (2001) MrBayes: Bayesian inference of phylogenetic trees. Bioinformatics 17: 754-755.

48. Larget B, Simon DL (1999) Markov chain Monte Carlo algorithms for the Bayesian analysis of phylogenetic trees. Mol Biol Evol 16: 750-759.

49. Sukosd Z, Knudsen B, Kjems J, Pedersen GNS (2012) PPfold 3.0: Fast RNA secondary structure prediction using phylogeny and auxiliary data. Bioinformatics 28: 2691-2692.

50. Zuker M (2003) Mfold web server for nucleic acid folding and hybridization prediction. Nucleic Acids Res 31: 3406-3415.

51. Darty K, Denise A, Ponty Y (2009) VARNA: Interactive drawing and editing of the RNA secondary structure. Bioinformatics 25: 1974-1975.

52. Koetschan C, Förster F, Keller A, Schleicher T, Ruderisch B, et al. (2010) The ITS2 Database III-sequences and structures for phylogeny. Nucleic Acids Research 38: D275-D279.

53. Leontis NB, Stombaugh J, Westhof E (2002) The non-Watson-Crick base pairs and their associated isostericity matrices. Nucleic Acids Res 30: 3497-3531.

54. Stombaugh J, Zirbel CL, Westhof E, Leontis NB (2009) Frequency and isostericity of RNA base pairs. Nucleic Acids Res 37: 2294-2312.

55. Wrona B, Grabowski M (2004) Etiology of apple sooty blotch in Poland. J Plant Protection Res 44: 294-297.

56. Zhuang J, Zhu M, Zhang M, Lin H, Lei Y, et al. (2010) Phialophora sessilis, a species causing flyspeck signs on bamboo in China. Mycotaxon 113: 405-413.

57. Sutton BC (1975) Hyphomycetes on cupules of Castanea sativa. Trans Brit Mycol Soc 64: 405-426

58. Hoog GS de, Hogeweg P, Meuzelaar HLC, Weijman ACM (1977) Rhinocladiella and allied genera. Stud Mycol 15: 1-136.

59. Perfect JR, Schell WA (1996) The new fungal opportunists are coming. Clin Infect Dis 22 (Suppl. 2): 8112-8118.

60. Hamada N, Abe N (2010) Growth characteristics of four fungal species in bathrooms. Biocontrol Sci 15: 111-115.

61. Lian X, Hoog GS de (2010) Indoor wet cells harbour melanized agents of cutaneous infection. Med Mycol 48: 622-628.

62. Summerbell R, Cooper E, Bunn U, Jamieson F, Gupta AK (2005) Onychomycosis: a critical study of techniques and criteria for confirming the etiologic significance of nondermatophytes. Medical Mycology 43: 39-59.
63. Nag Raj TR (1977) Ypsilonia, Acanthotheciella, and Kazulia gen. nov. Can J Bot 55: 1599-1622.

64. Hughes SJ (1976) Sooty molds. Mycologia 68: 693-820

65. Constantinescu O, Holm K, Holm L (1989) Teleomorph-anamorph connections in Ascomycetes. 1-3. Stanhughesia (Hyphomycetes) new genus, the anamorph of Ceramothyrium. Stud Mycol 31: 71-84.

66. Fuckel L (1874) Symbolae Mycologicae. Zweiter Nachtrag. Jahrbücher des Nassauischen Vereins für Naturkunde 27(28): 1-99.

67. Hawksworth DL (1980) Notes on some fungi occurring on Peltigera, with a key to accepted species. Trans Br Mycol Soc 74: 363-386.

68. Untereiner WA, Gueidan C, Orr M-J, Diederich P (2011) The phylogenetic position of the lichenicolous ascomycete Capronia peltigerae. Fung Diver 49: 225

69. Crous PW, Groenewald JZ, Summerell B (2007) Exophiala placitae Crous \& Summerell, sp. nov. Fungal Planet, 17. Centraalbureau voor Schimmelcultures, Utrecht, Netherlands.

70. Hibbett DS, Binder M, Bischoff JF, Blackwell M, Cannon PF, et al. (2007) A higher-level phylogenetic classification of the Fungi. Mycol Res 111: 509-547.

71. Barr ME (1976) Perspectives in the Ascomycotina. Mem. New York Bot. Gard. 28: $1-8$

72. Barr ME (1987) Prodromus to class Loculoascomycetes. Published by the author. Amherst, Massachusetts. $168 \mathrm{p}$

73. Winka K, Eriksson OE, Bång $\AA$ (1998) Molecular evidence for recognizing the Chaetothyriales. Mycologia 90: 822-830.

74. Kirk PM, Cannon PF, Minter DW, Stalpers JA (2008). Dictionary of the Fungi (10th ed). Wallingford, UK: CABI. $446 \mathrm{p}$

75. Untereiner WA, Naveau F (1999) Molecular systematics of the Herpotrichiellaceae with an assessment of the phylogenetic positions of Exophiala dermatitidis and Phialophora americana. Mycologia 91: 67-83.

76. Untereiner WA (2000) Capronia and its anamorphs: exploring the value of morphological and molecular characters in the systematics of the Herpotrichiellaceae. Stud Mycol 45: 141-148.

77. Gueidan G, Ruibal CV, de Hoog GS, Gorbushina AA, Untereiner WA, et al. (2008) A rock-inhabiting ancestor for mutualistic and pathogen-rich fungal lineages. Stud Mycol 61: 111-119.

78. Schol-Schwarz MB (1968) Rhinocladiella, its synonym Fonsecaea and its relation to Phialophora. Anton Leeuw Int J G 34: 119-152.

79. Samuels GJ, Müller E (1978) Life-history studies of Brazilian ascomycetes. 3. Melanomma radicans sp. nov. and its Apiosphaeria anamorph, Trematosphaeria perrumpens sp. nov, and Berlesiella fungicola sp. nov. and its Ramichloridium anamorph. Sydowia 31: 142-156.

80. Müller E, Petrini O, Fisher PJ, Samuels GJ, Rossman AY (1987) Taxonomy and anamorphs of the Herpotrichiellaceae with notes on generic synonomy. Trans Br Mycol Soc 88: 63-74.

81. Untereiner WA (1995) Fruiting studies in species of Capronia (Herpotrichiellaceae). Anton Leeuw Int J G 68: 3-17.

82. Untereiner WA (1997) Taxonomy of selected members of the ascomycete genus Capronia with notes on anamorph-teleomorph connections. Mycologia 89: $120-131$.

83. Okada G, Seifert KA, Takematsu A, Yamaoka Y, Miyazaki S, et al. (1998) A molecular phylogenetic reappraisal of the Graphium complex based on $18 \mathrm{~S}$ rDNA sequences. Can J Bot 76: 1495-1506.

84. Cheewangkoon R, Groenewald JZ, Summerell BA, Hyde KD, To-anun C, et al. (2009) Myrtaceae, a cache of fungal biodiversity. Persoonia 23: 55-85.

85. Sterflinger K, De Baere R, de Hoog GS, De Wachter R, Krumbein WE, et al (1997) Coniosporium perforans and C. apollinis, two new rock-inhabiting fungi isolated from marble in the Sanctuary of Delos (Cyclades, Greece). Anton Leeuw Int J G 72: 349-363.

86. Sert HC, Sterflinger K (2010) A new Coniosporium species from historical marble monuments. Mycol Prog 9: 353-359.

87. Li DM, Hoog GS de, Lindhardt Saunte DM, Gerrits van den Ende AHG, Chen XR (2008) Coniosporium epidermidis sp. nov., a new species from human skin. Stud Mycol 61: 131-136.

88. Hutchison LJ, Untereiner WA, Hiratsuka Y (1995) Knufia cryptophialidica gen. et sp. nov., a dematiaceous hyphomycete isolated from black galls of trembling aspen (Populus tremuloides). Mycologia 87: 902-908.

89. Coleman AW, Maria Preparata R, Mehrotra B, Mai JC (1998) Derivation of the secondary structure of the ITS-1 transcript in Volvocales and its taxonomic correlations. Protist 149: 135-146.

90. Gottschling M, Hilger H, Wolf M, Diane N (2001) Secondary structure of the ITS1 transcript and its application in a reconstruction of the phylogeny of Boraginales. Plant Biol 3: 629-636.

91. Goertzen LR, Can JJ, Gutell RR, Jasen RK (2003) ITS secondary structure derived from comparative analysis: implications for sequence alignment and phylogeny of the Asteraceae. Mol Phylogenet Evol 29: 216-234.

92. Thornhill DJ, Lord JB (2010) Secondary structure models for the internal transcribed spacer (ITS) region 1 from symbiotic dinoflagellates. Protist 161: $434-451$.

93. Bridge PD, Schlitt T, Cannon PF, Buddie AG, Baker M, et al. (2008) Domain II hairpin structure in ITS1 sequences as an aid in differentiating recently evolved animal and plant pathogenic fungi. Mycopathologia 166: 1-16.

94. Rodriguez-Martínez R, Rocap G, Logares R, Romac S, Massana R (2012) Low evolutionary diversification in a widespread and abundant uncultured protist (MAST-4). Mol Biol Evol 29: 1393-1406. 
95. Réblová M, Winka K (2000) Phylogeny of Chaetosphaeria and its anamorphs based on morphological and molecular data. Mycologia 92: 939-954.

96. Ullrich B, Reinhold K, Niehuis O. Misof B (2010) Secondary structure and phylogenetic analysis of the internal transcribed spacers 1 and 2 of bush crickets (Orthoptera: Tettigoniidae: Barbitistini). J Zool Syst Evol Res 48: 219-228.

97. Caisová L, Marin B, Melkonian M (2011) A close-up view on ITS2 evolution and speciation - a case study in the Ulvophyceae (Chlorophyta, Viridiplantae). BMC Evol Biol 11: 262. Available: http://www.biomedcentral.com/14712148/11/262. Accessed 20 September 2011.

98. Schultz J, Maisel S, Gerlach D, Müller T, Wolf M (2005) A common core of secondary structure of the internal transcribed spacer 2 (ITS2) throughout the Eukaryota. RNA 11: 361-364.

99. Coleman AW, van Oppen MJH (2008) Secondary structure of the rRNA ITS2 region reveals key evolutionary patterns in acroporid corals. J Mol Evol 67: 389-396.

100. Gould IR, Kollman PA (1994) Theoretical Investigation of the Hydrogen Bond Strengths in Guanine-Cytosine and Adenine-Thymine Base Pairs. J Am Chem Soc 116: 2493-2499.

101. Sykes MT, Levitt M (2005) Describing RNA structure by libraries of clustered nucleotide doublets. J Mol Biol 351: 26-38.
102. Fabry S, Köhler A, Coleman AW (1999) Intraspecies analysis: comparison of ITS sequence data and gene intron sequence data with breeding data for a worldwide collection of Gonium pectorale. Journal of Molecular Evolution 48: 94 101 .

103. Coleman AW (2005) Paramecium aurelia revisited. J Eukaryot Microbiol 52: 6877.

104. Amato A, Kooistra WHCF, Ghiron JHL, Mann DG, Pröschold T, et al. (2007) Reproductive isolation among sympatric cryptic species in marine diatoms. Protist 158: 193-207.

105. Coleman AW (2003) ITS2 is a double-edged tool for eukaryote evolutionary comparisons. Trends Genet 19: 370-375.

106. Coleman AW, Vacquier VD (2002) Exploring the phylogenetic utility of ITS sequences for animals: a test case for abalone (Haliotis). J Mol Evol 54: 246-257.

107. Schultz J, Muller T, Achtziger M, Seibel P, Dandekar T, et al. (2006) The internal transcribed spacer 2 database-a web server for (not only) low level phylogenetic analyses. Nucleic Acids Res 34: W704.

108. Selig C, Wolf M, Muller T, Dandekar T, Schultz J (2007) The ITS2 Database II: homology modeling RNA structure for molecular systematics. Nucleic Acids Res 36: D377-D380.

109. Müller T, Philippi N, Dandekar T, Schultz J, Wolf M (2007). Distinguishing species. RNA 13: 1469. 